\title{
Are Targets for Renewable Portfolio Standards Too Low? The Impact of Market Structure on Energy Policy
}

\author{
Afzal S. Siddiqui* \\ Department of Statistical Science, University College London, UK and Department of Computer and \\ Systems Sciences, Stockholm University, Sweden \\ Makoto Tanaka \\ National Graduate Institute for Policy Studies (GRIPS), Tokyo, Japan \\ Yihsu Chen \\ Department of Technology Management, University of California Santa Cruz, Santa Cruz, CA, USA
}

\begin{abstract}
In order to limit climate change from greenhouse gas emissions, governments have introduced renewable portfolio standards (RPS) to incentivise renewable energy production. While the response of industry to exogenous RPS targets has been addressed in the literature, setting RPS targets from a policymaker's perspective has remained an open question. Using a bilevel model, we prove that the optimal RPS target for a perfectly competitive electricity industry is higher than that for a benchmark centrally planned one. Allowing for market power by the non-renewable energy sector within a deregulated industry lowers the RPS target vis-à-vis perfect competition. Moreover, to our surprise, social welfare under perfect competition with RPS is lower than that when the non-renewable energy sector exercises market power. In effect, by subsidising renewable energy and taxing the non-renewable sector, RPS represents an economic distortion that over-compensates damage from emissions. Thus, perfect competition with RPS results in "too much" renewable energy output, whereas the market power of the non-renewable energy sector mitigates this distortion, albeit at the cost of lower consumer surplus and higher emissions. Hence, ignoring the interaction between RPS requirements and the market structure could lead to sub-optimal RPS targets and substantial welfare losses.

Keywords: OR in environment and climate change, renewable portfolio standards, bi-level modelling, market power
\end{abstract}

\footnotetext{
*Corresponding author

Email address: afzal.siddiqui@ucl.ac.uk (Afzal S. Siddiqui*)
} 


\section{Introduction}

Concerns about climate change have spurred policymakers to implement measures to reduce greenhouse gas emissions. One of the challenges faced by regulators is to craft policies that are compatible with profit maximisation and to allow polluting industries to reduce emissions in a competitive market. Two commonly implemented policy instruments are cap-andtrade $(\mathrm{C} \& \mathrm{~T})$ schemes and policies for promoting renewable energy (RE) technologies. While the former directly limit GHG emissions, the latter do so indirectly by providing financial incentives in the form of either subsidies or guaranteed revenue for RE producers. Examples of C\&T schemes are the EU Emissions Trading System (ETS), the Regional Greenhouse Gas Initiative (RGGI), and California Assembly Bill (AB) 32. On the other hand, support for RE adoption includes feed-in tariffs (FiT) and renewable portfolio standards (RPS). A FiT allows producers to lock in future revenue through guaranteed prices for power generated from RE sources, which provides access to capital on better terms for financing projects. By contrast, RPS requires a certain percentage (or, a MWh amount in some U.S. states) of electricity generation to originate from RE sources. Examples include RPS policies in various U.S. states as well as the EU 20-20-20 targets (European Commission, 2009). In most cases, producers are allowed to meet RPS by self-generation, procuring power from RE sources via bi-lateral contracts, and purchasing RE certificates/credits (RECs) from secondary markets. The main difference between these two instruments is that while FiT is determined by negotiation between RE producers and the government, the REC price is endogenously determined by the supply-demand condition in the REC market. See Murphy and Rosenthal (2006) for an approach to quantifying the marginal contribution of a given policy measure that is included in a portfolio of several measures.

RPS has received some attention in the literature partly due to its popularity in the U.S. and other countries. In contrast to the extant literature, we take a policy perspective to explore the unintended consequences of a policymaker's failure to account for market structure when setting the RPS target. To our surprise, we find that the RPS policy may actually perform better under some market power. Early studies on RPS are mostly qualitative, focusing on description and explanation of RPS or comparison of RPS implementations among U.S. states (Bird and Lokey, 2007; Holt and Wiser, 2007; Wiser and Barbose, 2008). There is a handful of studies empirically identifying the impact of RPS on RE capacity. For example, cross-sectional analyses are performed by Menz and Vachon (2006) and Adelaja and Hailu (2008), and both conclude that a positive (not causal) relationship exists between RPS and RE capacity. Using panel data design, Kneifel (2008) concludes that RPS resulted in no statistically meaningful increase in RE capacity. However, when accounting for differences in RPS coverage (i.e., among public utilities, investor-owned utilities, and power marketers, etc., 
as well as existing capacity), Yin and Powers (2010) find that RPS spurred RE development.

A number of papers on RPS have focused on analyses of industry response or design of RPS. For example, Boomsma et al. (2012) use real options to explore the response of a power company facing exogenous stochastic REC prices. Treating the REC price as endogenous, Chen and Wang (2013) examine the interactions among RPS, emissions trading, and green energy programs by considering two aspects of RPS policy design: double-counting and bundling. The theoretical analysis shows that bundling could be redundant in the presence of double-counting, which refers to the use of renewable energy both to satisfy the RPS requirement (or to sell energy to other parties in form of RECs) and to sell energy to consumers as green power to earn a premium. Policies that allow for double-counting appear to be a better choice since they result in a higher social surplus. On a related note, Chen et al. (2011) tackle via a complementarity model whether restrictions imposed on instate or out-of-state sales for the purposes of curtailing emissions in line with California's AB 92 would produce the same pattern of electricity generation. Amundsen and Mortensen (2001) investigate REC and other tradeable permit markets via an equilibrium model in the context of Danish legislation. Fischer (2010) explores the impact of RPS on the primary market, i.e., the equilibrium electricity price. She finds that modest RPS targets may initially lower the equilibrium electricity price because the REC price serves as a subsidy for RE. However, more stringent RPS targets cause non-renewable (NRE) production to reduce drastically, thereby putting upward pressure on the equilibrium electricity price and providing consumers with a strong signal to reduce demand. By allowing for producers to behave à la Cournot, Amundsen and Bergman (2012) show that the exercise of market power in the presence of green certificates could have deleterious effects on social welfare by stymieing RE producers. Tanaka and Chen (2013) extend this analysis to allow for the exercise of market power by a dominant producer acting as a Stackelberg leader. The resulting mathematical program with equilibrium constraints (MPEC) illustrates how an NRE producer with market power can manipulate prices in both the primary and secondary markets with adverse consequences for RE investors. Finally, Hibiki and Kurakawa (2013) consider a dominant-fringe framework to compare social welfare under FiT and RPS. They find that FiT can achieve higher social welfare than RPS when the marginal damage cost is relatively low.

Policy debate over the RPS target has received little attention partially because it is perceived that the process is more akin to political negotiation rather than something grounded in economic principles, i.e., as a result of equating marginal damage costs to marginal abatement costs. This is a juncture that marks a point of departure between our paper and extant work. Specifically, our paper focuses on comparing second-best RPS policies in a decentralised industry with a first-best choice implemented by an idealised central planner. 
Towards this end, we assume that the decentralised settings have a policymaker that sets an RPS target, while energy producers are followers. Our main objective is to compare optimal RPS targets and to understand producers' incentives under such policies as well as to gauge their implications for social welfare. We, thus, consider three different settings by analysing their solutions: (1) a (first-best) benchmark setting, in which a central planner controls all aspects of the energy sector by maximising social welfare inclusive of the cost of damage from emissions; (2) a perfectly competitive decentralised setting, in which generation decisions at the lower level are made by price-taking NRE and RE sectors who take the RPS target as well as the REC price as given, while a welfare-maximising policymaker at the upper level determines the optimal RPS target; and finally (3) a decentralised setting as in (2) but with an NRE sector that is allowed to exercise market power by behaving $\grave{a}$ la Cournot.

With its treatment of strategic interactions among distinct agents via a multi-level model, our paper is similar in spirit to Sauma and Oren (2006) and Murphy and Smeers (2010). Sauma and Oren (2006) assess transmission line investment by formulating the problem as an equilibrium problem with equilibrium constraints (EPEC) with each producer investing in generation capacity at an upper level and market clearing at the lower level comprising producers' generation and the system operator's re-dispatching decisions. A welfare-maximising social planner uses this EPEC to evaluate various transmission expansion proposals. Their results highlight the importance of accounting for market structure when assessing transmission investment. To put it differently, a proactive social planner who explicitly considers the response of strategic producers can lead to a better outcome as measured by social surplus than a reactive planner who ignores generation capacity addition. Meanwhile, Murphy and Smeers (2010) analytically explore capacity investments, forward contracting, and spot market dispatch in a duopoly. Their resulting tri-level model, which is more complicated than an EPEC, is solved using a stylised representation of the electricity industry, i.e., constant marginal costs and no transmission constraints. The authors find that the classical result of Allaz and Vila (1993), i.e., that forward contracts may mitigate market power, may not hold when capacity investments are required.

In a similar vein, we aim to derive closed-form solutions to a multi-level model but with the intention of examining how policy may be designed. In particular, we formalise analytically how the very nature of the energy sector's equilibrium is affected when another decisionmaking level is considered. Indeed, in decentralised settings, since the cost of the externality from emissions is not directly borne by producers, their private incentives have to be aligned with those of society via the RPS. We prove all of our results analytically by finding quasiclosed-form solutions for bi-level models. To best of our knowledge, this is the first paper that studies the endogenous setting of the RPS target from a policymaker's perspective by 
considering the market structure with tradeable RECs.

The rest of this paper is organised as follows. Section 2 lays out the modelling assumptions. We develop the main mathematical model and derive analytical results in Section 3 for each type of market setting. Section 4 uses numerical examples to illustrate the insights, while Section 5 summarises the work and offers directions for future research. All proofs of the propositions may be found in the appendix.

\section{Assumptions}

\section{Parameters}

A Intercept of the inverse demand function $(\$ / \mathrm{MWh})$

$C_{n} \quad$ Cost of NRE production $(\$ / \mathrm{MWh})$

$C_{r} \quad$ Cost of RE production $(\$ / \mathrm{MWh})$

$K \quad$ Rate of increase in marginal cost of greenhouse gas emissions $\left(\$ / \mathrm{MWh}^{2}\right)$

Z Slope of inverse demand function $\left(\$ / \mathrm{MWh}^{2}\right)$

\section{Primal Variables}

$\alpha \quad$ Optimal proportion of electricity from RE (-)

$q_{n} \quad$ NRE production (MWh)

$q_{r} \quad$ RE production (MWh)

$q \quad$ Total electricity consumption (MWh)

\section{Dual Variables}

$p^{R E C} \quad$ Market-clearing price for RECs $(\$ / \mathrm{MWh})$

We assume that there are two types of generators in the electricity industry: renewable and non-renewable. Since we are interested in industry-wide effects, we aggregate these two types of generators into sectors. Thus, effectively, we have distinct RE and NRE sectors in the industry, each of which acts as if it were a conglomerate. For each sector, we assume linear cost functions, $c_{n}\left(q_{n}\right)=C_{n} q_{n}$ and $c_{r}\left(q_{r}\right)=C_{r} q_{r}$, which reflect not only marginal costs of generation but also amortised capital costs. Here, $q_{n}$ and $q_{r}$ are NRE and RE production (in MWh), respectively. Although non-linear cost functions may be more realistic in terms of capturing the effects of start-up costs, ramping constraints, and capacity limits, 
our aggregated treatment of the two sectors means that such attributes are less relevant than in a firm-level analysis.

As for the demand side, we also aggregate it by representing consumers' willingness to pay as a linear inverse demand function, i.e., $p(q)=A-Z q$ (in $\$ / \mathrm{MWh}$ ), where $q=q_{n}+q_{r}$ is total consumption. We assume that $A>C_{r}>C_{n}$ in order to ensure that there is an equilibrium and to capture the general characteristic that RE has higher levelised costs than NRE sources. Here, $A>0$ (in $\$ / \mathrm{MWh}$ ) and $Z>0$ (in $\left.\$ / \mathrm{MWh}^{2}\right)$ are the intercept and slope of the inverse demand function, respectively. The externality from emissions is included via a damage function that is convex in only the generation from the NRE sector, i.e., $d\left(q_{n}\right)=\frac{1}{2} K q_{n}^{2}$ for $K>0$. Here, $K$ (in $\$ / \mathrm{MWh}^{2}$ ) is the rate of increase in marginal cost of emissions. Other types of damage functions may be posited, but increasing marginal effects from emissions capture the fact that atmospheric concentrations of greenhouse gases are more difficult to reverse the greater that they are. In order to facilitate comparative statics of the resulting solutions, we assume that $K>Z$.

We take a complementarity approach to model the interaction between a deregulated electricity industry and a policymaker by assuming that the policymaker's objective is to maximise social welfare (SW) inclusive of damage costs. In order to explore the variation of outcomes, we allow for the following market settings:

Central planning (CP) This benchmark setting has a central planner operating all power plants in order to maximise SW considering damage from emissions. This results in a single-level quadratic program (QP).

Perfect competition (PC) Generation decisions at the lower level are made by pricetaking RE and NRE sectors who take the RPS percentage target, $\alpha$, as given and maximise their profits inclusive of REC revenues or costs determined by $p^{R E C}$, the shadow price of the RPS constraint. At the upper level, the policymaker sets the RPS percentage in order maximise SW constrained by the lower-level mixed complementarity problem (MCP). This bi-level program may be re-cast as an MPEC if each lowerlevel problem is convex and may, thus, be replaced by its Karush-Kuhn-Tucker (KKT) conditions (Gabriel et al., 2012; Zhou et al., 2011).

Cournot oligopoly (CO) This is the same as PC except that the NRE sector is dominant and behaves à la Cournot, i.e., it is able to influence the electricity price, whereas the $\mathrm{RE}$ sector remains a price taker.

Since we will examine three market structures, we denote ${ }^{-}, \hat{\bullet}$, and $\cdot^{*}$ as the optimal values for decision variables in $\mathrm{CP}, \mathrm{PC}$, and $\mathrm{CO}$ settings, respectively. Finally, all decisions are made for a representative time period without uncertainty in any underlying parameter values. 


\section{Mathematical Model and Analytical Results}

\subsection{Central Planning}

The CP selects generation of either type in order to maximise SW by solving the following QP:

$$
\max _{q_{n} \geq 0, q_{r} \geq 0} \int_{0}^{q_{n}+q_{r}} p\left(q^{\prime}\right) d q^{\prime}-c_{n}\left(q_{n}\right)-c_{r}\left(q_{r}\right)-d\left(q_{n}\right)
$$

The KKT conditions for the problem in Eq. (11) are:

$$
\begin{array}{r}
0 \leq q_{n} \perp-A+Z\left(q_{n}+q_{r}\right)+C_{n}+K q_{n} \geq 0 \\
0 \leq q_{r} \perp-A+Z\left(q_{n}+q_{r}\right)+C_{r} \geq 0
\end{array}
$$

Effectively, the CP internalises the cost of emissions, i.e., each sector produces up to the point where its marginal revenue equals the marginal social cost, and the lack of REC revenues deters RE production.

Because the CP's QP is concave, globally optimal interior solutions may be obtained for output and the equilibrium electricity price:

$$
\begin{array}{r}
\bar{q}_{n}=\frac{\left(C_{r}-C_{n}\right)}{K} \\
\bar{q}_{r}=\frac{\left[A-C_{r}-\frac{Z}{K}\left(C_{r}-C_{n}\right)\right]}{Z} \\
\bar{q}=\frac{A-C_{r}}{Z} \\
\bar{p}=C_{r}=C_{n}+K \bar{q}_{n}
\end{array}
$$

The fraction of electricity from renewable sources under CP may be determined ex post as:

$$
\bar{\alpha} \equiv \frac{\bar{q}_{r}}{\bar{q}}=\frac{\left[A-C_{r}-\frac{Z}{K}\left(C_{r}-C_{n}\right)\right]}{\left(A-C_{r}\right)}
$$

Proposition 1. Under an interior solution for $C P$, ceteris paribus increases in $K$ result in:

(i) A decrease in the optimal NRE output.

(ii) An increase in the optimal RE output.

(iii) No change in the equilibrium electricity consumption.

(iv) No change in the equilibrium electricity price. 


\section{(v) An increase in the proportion of electricity from $R E$.}

Parts (i), (ii), and (v) of Proposition 1 are intuitive: greater marginal cost of damage from emissions will induce the CP to substitute NRE output with RE output. However, parts (iii) and (iv) are somewhat counterintuitive because one would expect consumption to decrease as a result of higher marginal costs of damage from emissions. In fact, what happens is that as long as $q_{r}>0$, the KKT conditions for optimality effectively equate the marginal costs of output from RE and NRE sources, i.e., $C_{r}=C_{n}+K q_{n}$. Therefore, this relationship fixes the equilibrium output, and further increases to $K$ merely change the composition of output. By contrast, as long as $K$ is relatively low, i.e., $C_{r}>C_{n}+K q_{n}$, we have the following corner solution in which the equilibrium energy output decreases with $K$ :

$$
\begin{array}{r}
\bar{q}_{n}^{0}=\frac{\left(A-C_{n}\right)}{K+Z} \\
\bar{q}_{r}^{0}=0 \\
\bar{q}^{0}=\frac{\left(A-C_{n}\right)}{K+Z} \\
\bar{p}^{0}=\frac{\left(A K+Z C_{n}\right)}{K+Z}
\end{array}
$$

As for the equilibrium price, it is simply equal to the $\mathrm{RE}$ generation cost for an interior solution, which is unaffected by $K$. In the event of a corner solution, the equilibrium price increases with $K$ to reflect the reduction in consumption required. In summary, with CP, when $K$ is relatively low, the optimal decision is to reduce consumption by curbing NRE production as $K$ increases without relying on RE sources. Once $K$ is high enough to equate the incremental cost of RE production to the incremental social cost of NRE output, it is optimal to fix the total energy output (and, therefore, the equilibrium price) and to respond to further increases in $K$ by merely changing the mix of electricity generation.

Before moving on to settings with deregulated industries, it will be helpful to note that $\bar{\alpha}$ is the root of the following linear function:

$$
\overline{\mathcal{Q}}(\alpha, K)=-K\left(A-C_{r}\right) \alpha-Z\left(C_{r}-C_{n}\right)+K\left(A-C_{r}\right)
$$

Assuming $\overline{\mathcal{Q}}(0, K)>0$ guarantees that an interior solution exists and is unique because $\overline{\mathcal{Q}}(1, K)=-Z\left(C_{r}-C_{n}\right)<0$.

\subsection{Perfect Competition}

In a deregulated setting, each sector seeks to maximise its own profit at the lower level by selecting the level of output taking into account the RPS set by the policymaker at the upper 
level. No sector directly faces the cost of damage from emissions, but the electricity industry on aggregate is subject to the RPS constraint, which may impose an effective tax on the NRE sector. We start with a case of perfect competition in which both NRE and RE sectors are price takers. The bi-level problem is solved via backward induction by first tackling the lower level, which can be re-cast as an MCP because each lower-level problem is convex. The resulting MCP is parameterised by the upper-level's RPS decision. Next, the bi-level problem is re-cast as an MPEC by replacing the lower level by the parameterised MCP, which defines the MPEC's feasible set together with the upper-level constraint. Finally, assuming interior solutions, we replace the MPEC by an unconstrained non-linear program (NLP).

\subsubsection{Lower-Level MCP with Perfect Competition}

At the lower level, each sector selects its production in order to maximise its profit, which consists of revenues from electricity sales minus production costs. For the NRE sector, the RPS requirement results in an extra cost resulting from the obligation to purchase RECs at the equilibrium REC price, $p^{R E C}$ (in $\$ / \mathrm{MWh}$ ). By contrast, the RE sector collects revenues from RECs in proportion to $p^{R E C}$ and the RPS constraint. Since each of these problems is convex, it may be replaced by its KKT conditions. Hence, the lower-level MCP in Eqs. (14) -(16) consists of each sector's KKT conditions for profit maximisation and the RPS constraint:

$$
\begin{array}{cl}
\max _{q_{n} \geq 0} \quad p q_{n}-c_{n}\left(q_{n}\right)-\alpha p^{R E C} q_{n} \\
\Rightarrow \quad 0 \leq q_{n} \perp-A+Z\left(q_{n}+q_{r}\right)+C_{n}+\alpha p^{R E C} \geq 0 \\
\max _{q_{r} \geq 0} \quad p q_{r}-c_{r}\left(q_{r}\right)+(1-\alpha) p^{R E C} q_{r} \\
\Rightarrow \quad 0 \leq q_{r} \perp-A+Z\left(q_{n}+q_{r}\right)+C_{r}-(1-\alpha) p^{R E C} \geq 0 \\
\quad 0 \leq p^{R E C} \perp q_{r}-\alpha\left(q_{n}+q_{r}\right) \geq 0
\end{array}
$$

For a given $\alpha$, the analytical interior solution to the MCP in Eqs. (14)-(16) is:

$$
\begin{gathered}
\hat{q}_{n}(\alpha)=\frac{(1-\alpha)\left[A-\left(\alpha C_{r}+(1-\alpha) C_{n}\right)\right]}{Z} \\
\hat{q}_{r}(\alpha)=\frac{\alpha\left[A-\left(\alpha C_{r}+(1-\alpha) C_{n}\right)\right]}{Z} \\
\hat{p}^{R E C}(\alpha)=C_{r}-C_{n} \\
\hat{p}(\alpha)=\alpha C_{r}+(1-\alpha) C_{n}
\end{gathered}
$$

Eqs. (19) and (20) indicate that the equilibrium REC price is simply the additional cost 
of $\mathrm{RE}$ production and the equilibrium electricity price is a weighted average of the two types of production costs, respectively. The following proposition summarises the impact of the RPS target on these lower-level decisions:

Proposition 2. Under an interior solution for the lower level of the $P C$, ceteris paribus increases in the RPS target result in:

(i) A decrease in the optimal NRE output.

(ii) Either an increase or a decrease in the optimal RE output.

(iii) A decrease in the equilibrium electricity consumption.

(iv) No change in the equilibrium REC price.

(v) An increase in the equilibrium electricity price.

Intuitively, parts (i)-(iii) of Proposition 2 state that as the RPS target increases, total quantity demanded decreases. However, while NRE production decreases monotonically with $\alpha$, the impact of RPS on RE production is ambiguous because it reduces overall production while increasing RE's share. By contrast, increasing the RPS target decreases not only total production but also NRE's share. Consequently, RE production first increases and then decreases with the RPS target. Finally, the equilibrium electricity price needs to increase at a rate of $C_{r}-C_{n}$ with $\alpha$ in order to match the required subsidy to the RE sector.

\subsubsection{Upper Level with Perfect Competition}

If the lower-level problems are replaced by their KKT conditions, then the policymaker's bi-level problem may be re-cast as an MPEC to select the optimal RPS, $\hat{\alpha}$, in order to maximise social welfare:

$$
\begin{array}{cl}
\max _{\{0 \leq \alpha \leq 1\} \cup\left\{q_{n}, q_{r}\right\} \cup\left\{p^{R E C}\right\}} & \text { Eq. (1) } \\
\text { s.t. } & \text { Eqs. (14) }-(16)
\end{array}
$$

Assuming an interior solution for the lower-level MCP and that $0<\alpha<1$, we can further replace the MPEC with the following unconstrained NLP:

$$
\max _{\alpha} \frac{A \hat{q}_{n}(\alpha)}{(1-\alpha)}-\frac{Z\left(\hat{q}_{n}(\alpha)\right)^{2}}{2(1-\alpha)^{2}}-C_{n} \hat{q}_{n}(\alpha)-\frac{C_{r} \hat{q}_{n}(\alpha) \alpha}{(1-\alpha)}-\frac{K\left(\hat{q}_{n}(\alpha)\right)^{2}}{2}
$$

The KKT condition for the NLP in Eq. (21) is:

$$
\frac{\left\{\left(C_{n}-C_{r}\right)\left(Z-K(1-\alpha)^{2}\right)+K(1-\alpha)\left[A-\left(\alpha C_{r}+(1-\alpha) C_{n}\right)\right]\right\}}{\left[A-\left(\alpha C_{r}+(1-\alpha) C_{n}\right)\right]^{-1} Z^{2}}=0
$$


Since $A-\left(\alpha C_{r}+(1-\alpha) C_{n}\right)>0$, the only way for the KKT condition to be met is for the expression in the curly brackets to equal zero. This effectively means finding the roots of the characteristic quadratic $\hat{\mathcal{Q}}(\alpha, K)=W(K) \alpha^{2}+X(K) \alpha+Y(K)$, where:

$$
\begin{array}{r}
W(K)=2 K\left(C_{r}-C_{n}\right)>0 \\
X(K)=K\left[3\left(C_{n}-C_{r}\right)+\left(C_{n}-A\right)\right]<0 \\
Y(K)=\overline{\mathcal{Q}}(0, K)+2 K\left(C_{r}-C_{n}\right)>0
\end{array}
$$

Thus, $\hat{\mathcal{Q}}(\alpha, K)$ is a convex quadratic with the following properties:

1. $\hat{\mathcal{Q}}(0, K)=Y(K)>0$

2. $\hat{\mathcal{Q}}(1, K)=W(K)+X(K)+Y(K)=-Z\left(C_{r}-C_{n}\right)<0$

Consequently, we can conclude that $\hat{\mathcal{Q}}(\alpha, K)$ has two roots, $0<\alpha_{1}<1$ and $\alpha_{2}>1$ such that:

$$
\begin{aligned}
& \alpha_{1}=\frac{-X(K)-\sqrt{X(K)^{2}-4 W(K) Y(K)}}{2 W(K)} \\
& \alpha_{2}=\frac{-X(K)+\sqrt{X(K)^{2}-4 W(K) Y(K)}}{2 W(K)}
\end{aligned}
$$

The solution we seek is $\alpha_{1}$, which is now labeled as $\hat{\alpha}$.

Although comparative statics may be performed directly on $\hat{\alpha}$ with respect to $K$ using the closed-form solution in Eq. (26), it is less tedious to manipulate the characteristic quadratic that implicitly defines it, i.e., $\hat{\mathcal{Q}}(\hat{\alpha}, K)=W(K) \hat{\alpha}^{2}+X(K) \hat{\alpha}+Y(K)=0$ (see Fig. 1). Using this, we obtain the following:

Proposition 3. The optimal RPS target under PC increases with K, i.e., $\frac{d \hat{\alpha}}{d K}>0$.

Intuitively, a higher marginal cost of damage from emissions will lead the policymaker to tighten the RPS requirement. Unlike the CP setting, the policymaker has no scope to reduce consumption directly, but Proposition 2(iii) indicates that a higher $\alpha$ will indirectly lead to such an outcome.

Next, from the geometry of the solution, we obtain the relative ordering of the optimal RPS targets:

Proposition 4. The optimal RPS target under PC is greater than that obtained under CP, i.e., $\bar{\alpha}<\hat{\alpha}$. 
The illustration of the proof is in Fig. 1, the root of the solid line indicates $\bar{\alpha}$. Starting at $\alpha=1$, we can do a linear projection of $\hat{\mathcal{Q}}(\alpha, K)$ from the point $(1, \hat{\mathcal{Q}}(1, K))$. Such a projection is precisely $\overline{\mathcal{Q}}(\alpha, K)$. Due to the convexity of $\hat{\mathcal{Q}}(\alpha, K)$, the result follows. Intuitively, a higher RPS target is required under PC relative to $\mathrm{CP}$ because in a deregulated industry, it is not possible for the policymaker to curb consumption to the extent that the incremental cost of RE output matches the incremental social cost of NRE production. Instead, by creating a secondary market for RECs, the policymaker introduces a distortion in which "too much" RE output is used from a social welfare perspective. In effect, as long as the policymaker has no control over consumption, the only recourse to curb emissions is to incentivise RE production.

Using the same geometric properties, we can conclude the following technical aspects of the optimal RPS target under PC:

Proposition 5. The RPS target under PC, $\hat{\alpha} \in(0,1)$, exists and is a unique solution to the policymaker's problem in Eq. (21).

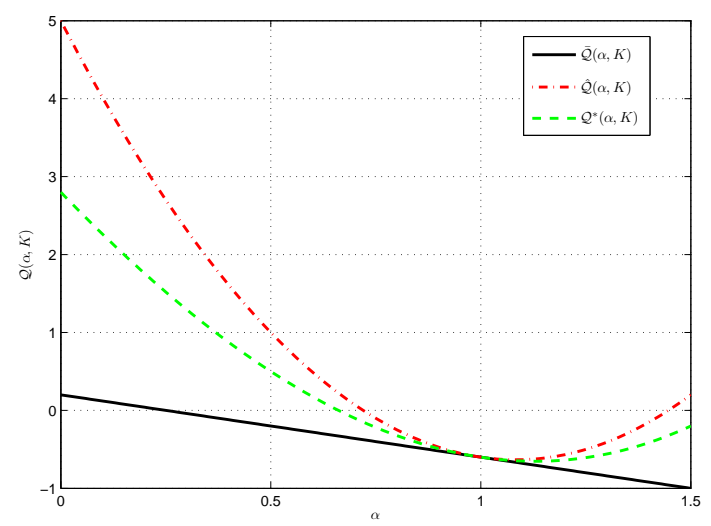

Figure 1: Characteristic Functions, $\overline{\mathcal{Q}}(\alpha, K), \hat{\mathcal{Q}}(\alpha, K)$, and $\mathcal{Q}^{*}(\alpha, K)$, for Representative Parameters

Other results pertaining to the impact of $K$ now follow:

Proposition 6. Under an interior solution for $P C$, ceteris paribus increases in $K$ result in:

(i) A decrease in the optimal NRE output.

(ii) Either an increase or a decrease in the optimal RE output.

(iii) A decrease in the equilibrium electricity consumption.

(iv) No change in the equilibrium REC price. 


\section{(v) An increase in the equilibrium electricity price.}

The intuition for Proposition 6 follows directly from Proposition 2 along with the monotonically increasing impact of the damage cost on the optimal RPS target under PC. It is worth contrasting the solution under PC with that under CP. Recall that with a corner solution under $\mathrm{CP}$, the equilibrium electricity consumption decreases along with a concomitant increase in the equilibrium electricity price even as no RE production occurs. In effect, for low relatively levels of $K$, it is optimal to curb consumption. Once RE production is marginal, further increases in the marginal damage cost lead to a change in the optimal production mix without affecting either the equilibrium electricity consumption or price. Lacking such direct control over the electricity industry, the policymaker in the PC setting can only indirectly influence outcomes by implementing more stringent RPS targets (Proposition 4).

\subsection{Cournot Oligopoly}

We now have the NRE sector behaving à la Cournot at the lower level with the RE sector still being a price taker. Again, the entire industry is subject to an RPS target imposed by the policymaker. However, since the NRE sector is able to manipulate the electricity price, such strategic behaviour will have implications for not only the industry equilibrium but also the optimal RPS target. Conceptually, the solution approach to the bi-level problem is the same as that in Section 3.2, i.e., replace the lower level by its KKT conditions to obtain an MPEC and then use the optimal interior solutions from the lower level to re-cast the MPEC as an NLP.

\subsubsection{Lower-Level MCP with Cournot Oligopoly}

At the lower level, the MCP still consists of the RE sector's KKT conditions for profit maximisation (Eq. (15)) and the RPS constraint (Eq. (16)). Since the NRE sector has market power, its KKT condition is modified to:

$$
\begin{array}{cl}
\max _{q_{n} \geq 0} & p\left(q_{n}+q_{r}\right) q_{n}-c_{n}\left(q_{n}\right)-\alpha p^{R E C} q_{n} \\
\Rightarrow & 0 \leq q_{n} \perp-A+Z\left(q_{n}+q_{r}\right)+Z q_{n}+C_{n}+\alpha p^{R E C} \geq 0
\end{array}
$$

Assuming $q_{n}>0, q_{r}>0$, and $p^{R E C}>0$, we can solve the MCP in Eqs. (15), (16), and (28) analytically (albeit as functions of $\alpha$ ):

$$
\begin{gathered}
q_{n}^{*}(\alpha)=\frac{(1-\alpha)\left[A-\left(\alpha C_{r}+(1-\alpha) C_{n}\right)\right]}{Z\left(\alpha^{2}-2 \alpha+2\right)} \\
q_{r}^{*}(\alpha)=\frac{\alpha\left[A-\left(\alpha C_{r}+(1-\alpha) C_{n}\right)\right]}{Z\left(\alpha^{2}-2 \alpha+2\right)}
\end{gathered}
$$




$$
\begin{gathered}
p^{R E C^{*}}(\alpha)=\frac{\left(C_{r}-C_{n}\right)-(1-\alpha)\left(A-C_{r}\right)}{\left(\alpha^{2}-2 \alpha+2\right)} \\
p^{*}(\alpha)=\frac{A(1-\alpha)^{2}+\alpha C_{r}+(1-\alpha) C_{n}}{\left(\alpha^{2}-2 \alpha+2\right)}
\end{gathered}
$$

Due to more complicated expressions, it is not as straightforward to do comparative statics with respect to the RPS level. However, at least these closed-form solutions may be manipulated further by defining $F(\alpha)=\alpha^{2}-2 \alpha+2$, which is greater than or equal to 1 for all values of $\alpha$, and $F^{\prime}(\alpha)=2(\alpha-1) \leq 0$ for all $0 \leq \alpha \leq 1$. Furthermore, it is possible to compare the equilibrium electricity and REC prices in the PC and CO settings for the same $\alpha$ :

Proposition 7. For a given RPS target, the equilibrium electricity price under $C O$ is higher than that under $P C$, i.e., $p^{*}(\alpha)>\hat{p}(\alpha)$.

Proposition 8. For a given RPS target, the equilibrium REC price under CO is lower than that under $P C$, i.e., $\hat{p}^{R E C}(\alpha)>p^{R E C^{*}}(\alpha)$.

Intuitively, by exercising market power, the NRE sector is able to increase the equilibrium electricity price and lower the equilibrium REC price to its benefit. As a consequence of market power, we also have the following results for NRE and RE outputs:

Proposition 9. For a given RPS target, the equilibrium NRE and RE outputs under CO are less than those under $P C$, i.e., $q_{n}{ }^{*}(\alpha)<\hat{q}_{n}(\alpha)$ and $q_{r}{ }^{*}(\alpha)<\hat{q}_{r}(\alpha)$.

\subsubsection{Upper Level with Cournot Oligopoly}

As before, the lower-level problems may be replaced by their KKT conditions, thereby rendering the policymaker's bi-level problem as an MPEC to select the optimal RPS, $\alpha$, in order to maximise social welfare:

$$
\begin{array}{cl}
\max _{\{0 \leq \alpha \leq 1\} \cup\left\{q_{n}, q_{r}\right\} \cup\left\{p^{R E C}\right\}} & \text { Eq. (1) } \\
\text { s.t. } & \text { Eqs. (15), (16) }) \text { (28) }
\end{array}
$$

Assuming that non-negativity constraints on the lower-level decision variables are satisfied with strict inequalities and that $0<\alpha<1$, we can replace the MPEC with the following unconstrained NLP:

$$
\max _{\alpha} \frac{A q_{n}^{*}(\alpha)}{(1-\alpha)}-\frac{Z\left(q_{n}^{*}(\alpha)\right)^{2}}{2(1-\alpha)^{2}}-C_{n} q_{n}^{*}(\alpha)-\frac{C_{r} q_{n}^{*}(\alpha) \alpha}{(1-\alpha)}-\frac{K\left(q_{n}^{*}(\alpha)\right)^{2}}{2}
$$


The KKT condition for the NLP in Eq. (33) is:

$$
\begin{aligned}
& \frac{\left[A-\left(\alpha C_{r}+(1-\alpha) C_{n}\right)\right]}{Z^{2} F(\alpha)^{3}}\left\{\left[Z(2 F(\alpha)-1)-K(1-\alpha)^{2}\right]\left(C_{n}-C_{r}\right) F(\alpha)\right. \\
& +\left[A-\left(\alpha C_{r}+(1-\alpha) C_{n}\right)\right] \times[F(\alpha)(K-2 Z)(1-\alpha) \\
& \left.\left.+2(1-\alpha)\left[Z(2 F(\alpha)-1)-K(1-\alpha)^{2}\right]\right]\right\}=0
\end{aligned}
$$

Since there is no apparent analytical solution to this KKT condition, we cannot derive the optimal RPS fraction as we did for the PC setting. However, as in the PC setting, we can manipulate the KKT condition to obtain more general insights about the behaviour of $\alpha^{*}$. We first note that the KKT condition in Eq. (34) holds as long as the expression in curly brackets is equal to zero because $\frac{\left[A-\left(\alpha C_{r}+(1-\alpha) C_{n}\right)\right]}{Z^{2} F(\alpha)^{3}}$ is strictly positive. Denoting the terms in the curly brackets of Eq. (34) as $\mathcal{Q}^{*}(\alpha, K)$, we can show that it is a cubic function whose shape depends on the sign of $K-2 Z$ since quartic terms cancel out. As shown in Fig. 1, the dashed curve intersects the horizontal axis from positive to negative exactly once for $0<\alpha<1$, thereby indicating that a solution for $\alpha^{*}$ exists and is unique. Unlike the PC setting, proving that $\alpha^{*}$ increases with the damage cost or is a unique optimum must be done indirectly:

Proposition 10. The RPS target under CO, $\alpha^{*} \in(0,1)$, exists and is a unique solution to the policymaker's problem in Eq. (33).

Proposition 11. The optimal RPS target under CO increases with K, i.e., $\frac{d \alpha^{*}}{d K}>0$.

Furthermore, we show that the maximised social welfare, denoted by $w$, has the following order:

Proposition 12. The maximised social welfare under $C O$ is greater than that for $P C$ but lower than that for $C P$, i.e., $\hat{w}<w^{*}<\bar{w}$.

Somewhat surprisingly, Proposition 12 indicates that PC results in the lowest maximised social welfare. First, based on the discussion related to Proposition 4 , the use of an RPS policy in a PC setting results in an economic inefficiency as "too much" RE production occurs vis-àvis CP. The mechanism behind this can be explained as follows. Under CP, efficient resource allocation can be achieved since the electricity price is equal to the marginal social cost, i.e., marginal production cost plus marginal damage cost or $\bar{p}=C_{r}=C_{n}+K \bar{q}_{n}$. By contrast, the policymaker can indirectly affect the market price only through the RPS target, $\alpha$, under PC. The electricity price is not equal to the marginal social cost as $\hat{p}(\alpha)=\alpha C_{r}+(1-\alpha) C_{n}<C_{r}$. This leads to inefficient resource allocation, whereby the RE sector supplies more than the 
efficient level. A CO setting also results in an economically inefficient level of NRE and RE output, but the NRE sector's exercise of market power (by withholding its output) puts downward pressure on RE output. While the decrease in total production and the resulting increase in the electricity price under $\mathrm{CO}$ have a negative effect on social welfare, the decrease in RE output has a positive impact on social welfare because it alleviates the distortion of resource allocation under PC. At the same time, the policymaker tries to mitigate the NRE sector's potential to exercise market power by curbing the RPS target relative to the PC level as shown in Proposition 13. Consequently, re-allocation of output from the RE sector to the NRE one occurs under $\mathrm{CO}$, which reduces the distortion under PC, thereby increasing social welfare.

Finally, and related to Proposition 12, it is possible to prove that the optimal RPS target has the following order:

Proposition 13. The optimal RPS target under CO is greater than that for CP but lower than that for $P C$, i.e., $\bar{\alpha}<\alpha^{*}<\hat{\alpha}$.

Intuitively, the policymaker mitigates the NRE sector's potential to exercise market power by curbing the RPS target relative to the PC level. Indeed, the NRE sector benefits from withholding generation, and a more stringent RPS target would play into its hands by facilitating the withholding of NRE production and increasing the equilibrium electricity price to the detriment of consumers. Conversely, the optimal RPS target under CO is lower bounded by the implied RPS target under CP.

\section{Numerical Examples}

\subsection{Data}

In order to gain further insights into the differences regarding the RPS targets and social welfare among the three settings, we implement numerical examples using the following parameter values: $A=100, Z=0.01, K \in[0.01,0.10], C_{n}=20$, and $C_{r}=80$. Note that we have set the renewable energy cost of production (inclusive of amortised capital costs) to be higher than that of non-renewable energy. We allow the damage cost parameter to vary in a range such that both corner and interior solutions are possible. For example, it is not until $K>0.03$ that the RE sector produces in all three settings. The case with $K=0$ is trivial because both central planning and perfect competition settings provide the same solution, i.e., $\bar{q}_{n}=8000, \bar{q}_{r}=0$, and $\bar{p}=20$, with a maximised social welfare of $\$ 320,000$ and zero profit for producers. Under Cournot oligopoly, $q_{n}^{*}=4000, q_{r}^{*}=0$, and $p^{*}=60$, and the NRE sector makes $\$ 160,000$ in profit with a maximised social welfare of $\$ 240,000$. In order to cope 
with corner and interior solutions, the MPECs corresponding to the PC and CO settings are implemented in GAMS with the NLPEC solver.

\subsection{Main Insights}

The impact of the damage from emissions on the RPS target is illustrated in Fig. 2, First, regardless of the setting, higher $K$ leads to increases in the RPS target, which was proven in Propositions 11(v), 3, and 11. Apart from $K \leq 0.03$ under the CP setting, the optimal response to increasing emissions costs involves supplanting NRE production by RE output. As noted in Eq. (9), for relatively low values of $K$, a corner solution is optimal under CP since NRE production is still more cost effective than RE even when internalising the cost of damage from emissions. Instead, the quantity demanded is reduced even as it continues to be satisfied exclusively by the NRE sector. By contrast, in the settings with deregulated electricity industries, the policymaker lacks control over generation and must rely on gradually increasing the RPS target to induce more RE production to mitigate higher costs of damage from emissions.

Second, following Propositions 4 and 13, the RPS target is highest under the PC setting followed by, in order, CO and CP. Somewhat counterintuitvely, the CP setting results in the lowest (implied) RPS target as the policymaker is able to internalise the cost of damage from emissions and issue drastic cuts in consumption. As such, RE output is not needed at all for relatively low levels of $K$ and is deployed in modest proportions thereafter. Lacking the scope to align private incentives of the sectors (to maximise profit) with societal ones (to maximise welfare), the policymaker in the PC and CO settings cannot rely as much on curbing consumption. Instead, it uses RECs to entice more RE production, thereby limiting the damage from emissions but not reducing total consumption by as much as in the CP setting. Thus, both the PC and CO settings result in a loss of welfare (as we shall explore later) relative to the $\mathrm{CP}$ setting. Comparing the $\mathrm{PC}$ and $\mathrm{CO}$ settings, the potential exercise of market power by the NRE sector in the latter prompts the policymaker to ease off on the RPS target.

The drivers for the dynamics of the optimal RPS targets also appear in the optimal NRE and RE production (Figs. 3 and 4). As demonstrated in Propositions 1(i) and 6(i), $\bar{q}_{n}$ and $\hat{q}_{n}$ decrease monotonically with $K$. Interestingly, in spite of the NRE sector's incentive to withhold generation in the CO setting, we actually observe $q_{n}^{*}>\hat{q}_{n}$. This outcome is a consequence of the fact that the policymaker in the $\mathrm{CO}$ setting recognises the potential for market power and scales back the RPS target relative to the PC setting. Indeed, if it were to set the RPS target in the CO setting equal to $\hat{\alpha}$, then the NRE sector would be more successful at raising the equilibrium electricity price and, thus, reducing consumer surplus. 


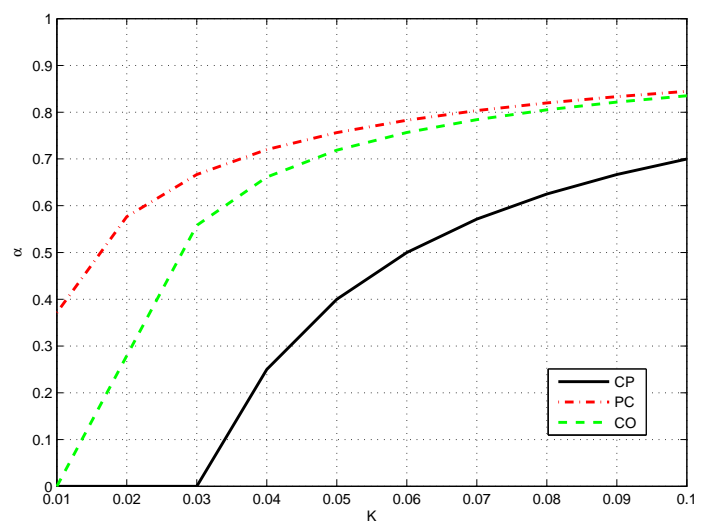

Figure 2: Optimal RPS Targets

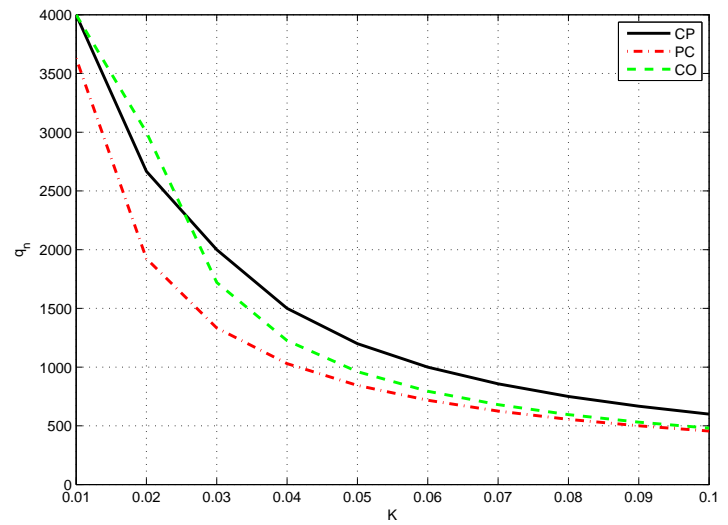

Figure 3: Optimal NRE Production

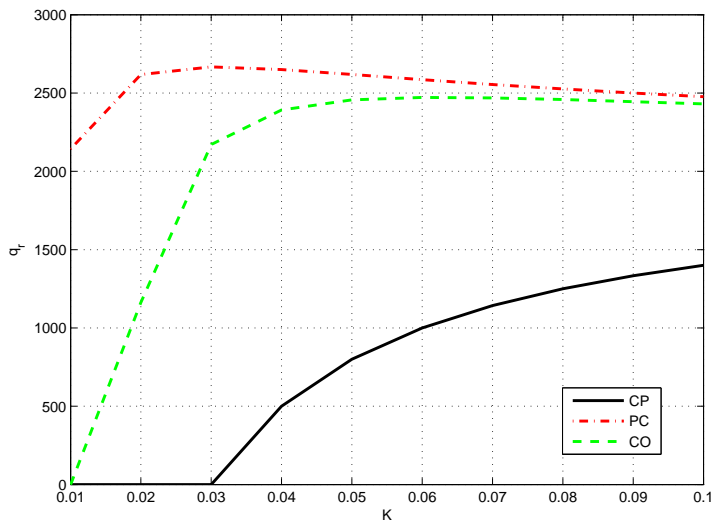

Figure 4: Optimal RE Production

Similarly, the impact of a higher cost of damage from emissions on RE production may be traced back to the optimal RPS targets. Under the CP setting, the RE sector becomes marginal once $K>0.03$, and we observe a steady increase in its output as indicated in Proposition 1(ii). In fact, there is a one-to-one correspondence between the rate of increase in RE production and the rate of decrease in NRE output. By contrast, under the PC setting, Proposition 6(ii) indicates that RE output may either increase or decrease with the damage cost of emissions. In Fig. 4, we observe that RE output first increases but then decreases. As noted in Fischer (2010), it becomes less costly to cut back on consumption when more stringent reductions in emissions are required rather than subsidising RE production. A similar pattern is observed in the CO setting, although the decline in RE production is more gentle as it was never subsidised as heavily as in the PC setting. Finally, it is worth noting that $\mathrm{RE}$ production is highest under the $\mathrm{PC}$ setting because of the economic inefficiency that a distortion such as REC introduces. Since the RPS target is lower under the CO setting, 
there is less of this distortion, and RE production is lowest under the CP setting because the marginal benefit of consumption is correctly matched with the marginal cost of production inclusive of all externalities from emissions.

In Fig. 5, we sketch out the behaviour of the equilibrium electricity price as the damage cost of emissions increases. First, as indicated in Proposition 1(iv), as long as the RE sector is marginal, further increases in $K$ do not affect $\bar{p}$ as the consumption does not change. Instead, only the production mix is altered to increase the proportion of RE output, and the equilibrium electricity price is simply the RE production cost. By contrast, when $K$ is low enough for $C_{r}>C_{n}+K q_{n}$ to hold, only NRE production is used, and reduction in emissions is attained via reduction in consumption. Consequently, there is an increase in the equilibrium electricity price only when a corner solution holds under CP. Next, since the RE sector is given an effective subsidy in the form of RECs under the PC setting, there is "too much" RE output from an efficiency perspective. This support for RE production (and tax on the NRE sector) enables a reduction in emissions without a drastic cut in consumption, which explains why the equilibrium electricity price is lower than under the CP setting. Nevertheless, an increase in the cost of damage from emissions leads to an increase in the equilibrium electricity price as indicated by Proposition 2(v). Under the CO setting, the equilibrium electricity price is higher than under the $\mathrm{PC}$ setting because of the exercise of market power by the NRE sector. However, because of the support for the RE sector, total consumption is higher than in the CP setting, thereby resulting in a lower equilibrium electricity price. Finally, the impact of $K$ on $p^{*}$ is non-monotonic: when the damage cost of emissions increases from 0.01 , there is actually a decrease in the equilibrium electricity price. This is a consequence of the NRE sector's withholding generation, which creates scope to increase total consumption once the RE sector is subsidised. Thus, a sufficient increase in $K$ means that the RE sector receives an increasing effective subsidy (see the REC price in Fig. 6), which causes total consumption to increase. In fact when $K$ increases from 0.01 to 0.02, total consumption increases from 4,000 MWh to 4,163 MWh. Subsequently, when $K$ increases to 0.03 , total consumption decreases to $3,892 \mathrm{MWh}$.

Turning to the REC price in Fig. 6, it remains constant at $C_{r}-C_{n}$ under the PC setting as shown in Eq. (19), as well as in Propositions 2(iv) and 6(iv). Effectively, there is a constant subsidy given to the RE sector funded by a constant tax on the NRE sector. However, in the $\mathrm{CO}$ setting, the REC price increases with the cost of damage from emissions. Again, this is because for low values of $K$, the NRE sector is able to exercise market power to boost the equilibrium electricity price and to depress the REC price. With an increasing cost of damage from emissions, the policymaker increases the RPS target, which ultimately dilutes the impact of the NRE sector's market power. Hence, in the limit, the REC price under CO 


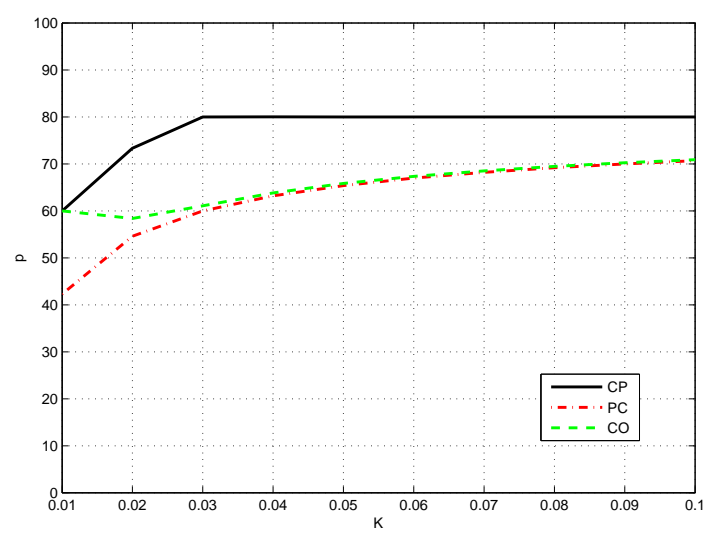

Figure 5: Equilibrium Electricity Price

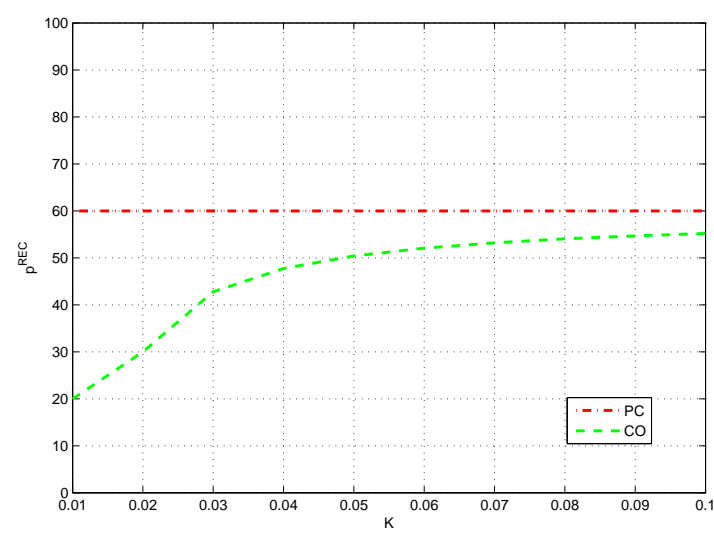

Figure 6: Equilibrium REC Price

converges to that under PC.

Recall that the policymaker in the $\mathrm{CP}$ setting curbs consumption heavily relative to the $\mathrm{PC}$ and $\mathrm{CO}$ settings. However, this means that the RE sector is not competitive with the NRE one unless the cost of damage from emissions increases sufficiently. While this results in the optimal solution from the perspective of social welfare (see Proposition 12), it, nevertheless, delivers the worst outcome in terms of the cost of damage from emissions (see Fig. 7). Conversely, the policymaker in the PC setting induces a greater reduction in emissions without extensively curbing consumption at the tradeoff of an economically inefficient transfer payment to the RE sector. The $\mathrm{CO}$ setting also results in a similar outcome, but the cost of damage from emissions is higher than under the PC setting as the policymaker refrains from mandating stringent RPS targets. Curiously, the cost of damage from emissions briefly increases for values of $K$ between 0.01 and 0.02 , which is because $K$ increases faster than the decrease in NRE output. Otherwise, in all settings, the total cost of damage from emissions decreases with $K$ as curtailment of NRE output has a stronger impact.

The crux of our policy findings is illustrated in Fig. 8, which reflects the order of the maximised social welfare derived in Proposition 12. Breakdowns of the consumer surplus and producer surplus are in Figs. 9 and 10, Recall that the CP setting delivers the socially optimal outcome as the externality from emissions is internalised: the social cost of NRE production increases with $K$, thereby resulting in a drastic reduction in consumption until RE production is competitive. After that point, consumption remains constant with only the generation mix shifting towards RE production. From an economic perspective, this is optimal because the most efficient resource mix is used to meet demand even though emissions remain relatively high. In effect, the $\mathrm{CP}$ implements a Pigouvian tax on emissions. Lacking such control over resource allocation, the policymaker in the PC and CO settings cannot 


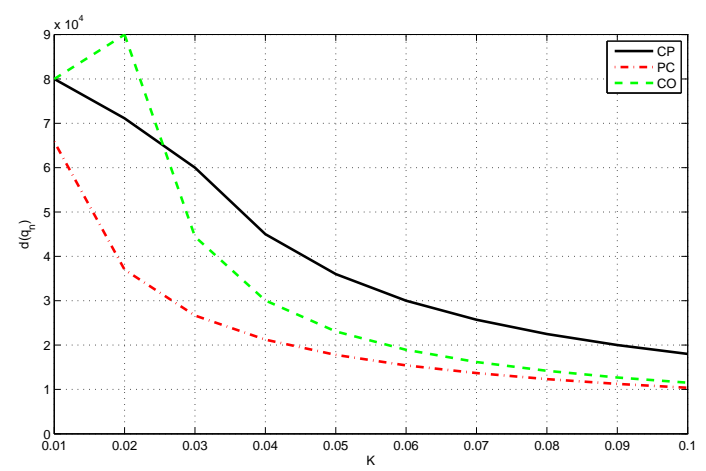

Figure 7: Cost of Damage from Emissions

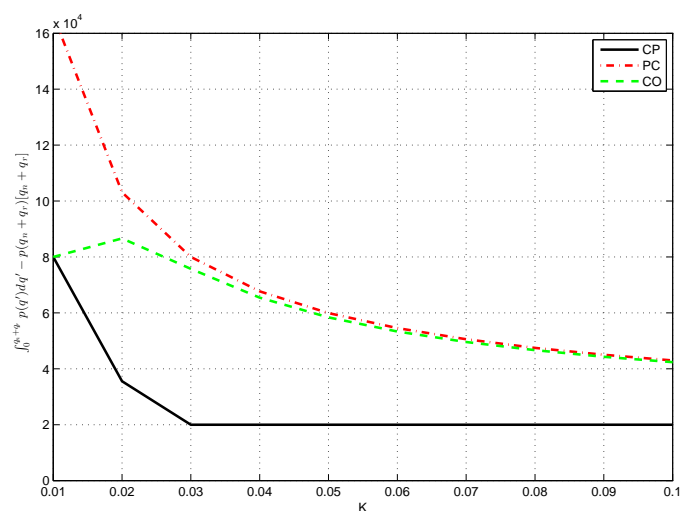

Figure 9: Consumer Surplus

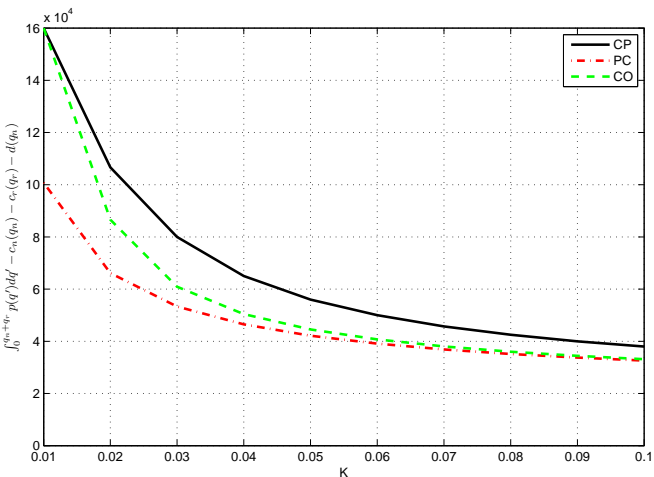

Figure 8: Maximised Social Welfare

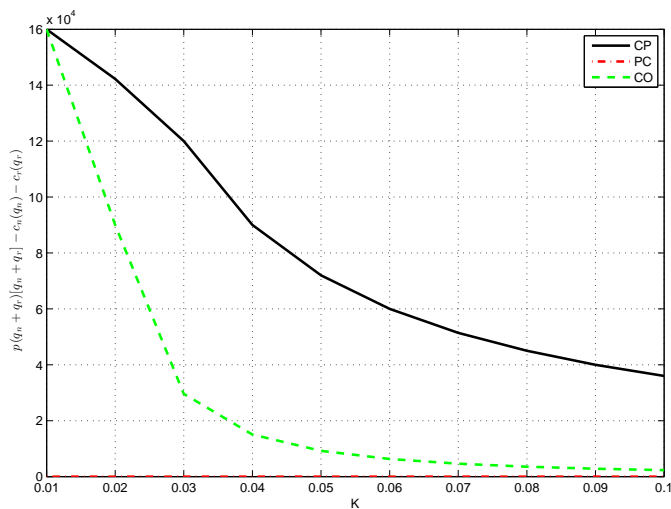

Figure 10: Producer Surplus

internalise the cost of damage from emissions. Indeed, curbing consumption or production is not directly possible. Instead, the policymaker supports the RE sector via REC payments, which are inefficient from an economic perspective as "too much" RE production occurs. Thus, this distortion mitigates the cost of damage from emissions while maintaining a high level of consumption but through economically inefficient transfer payments. Under the CO setting, social welfare somewhat paradoxically increases relative to the PC setting. This is because the exercise of market power by the NRE sector alleviates the distortionary effect of the RPS mandated by the policymaker. Hence, the economically inefficient RE production is pinned back, thereby leading to an increase in social welfare albeit at a cost to consumers (in the form of higher prices) and increased emissions.

Probing more into the ordering of the maximised social welfare, we plot the level curves of the welfare function in Fig. 11 for $K=0.04$. Under the CP setting, the optimal solution is $1500 \mathrm{MWh}$ and $500 \mathrm{MWh}$ from the NRE and RE sectors, respectively. Thus, the implied RPS level is $\bar{\alpha}=0.25$, and the maximised social welfare is $\$ 65,000$. As we move away from this point, we encounter lower iso-welfare contours. The binding RPS constraint through this 
point is $q_{r}=\left(\frac{\bar{\alpha}}{1-\bar{\alpha}}\right) q_{n}$, which has a slope of $\frac{1}{3}$ in this case. Now, if we fix the RPS target to 0.25 , then the lower-level MCPs in both the PC and CO settings will have equilibria that are defined by the intersections of the best-response functions of the NRE and RE sectors with the RPS constraint. However, these points will not be optimal for those settings. Indeed, allowing the RPS target to vary traces out the loci of equilibria for the lower-level MCPs, $\hat{g}\left(q_{n}\right)$ and $g^{*}\left(q_{n}\right)$, for PC and CO settings, respectively. The optimal solution for each of these deregulated settings is identified by a tangency condition between its respective locus and an iso-welfare contour. As can be seen from the figure, the locus of optimal solutions for the PC setting is always outside that of the one for the CO setting. Hence, its tangency with a level set of the social welfare function is at a lower value $(\$ 46,478$ in this case with $\left.\left(\hat{q}_{n}, \hat{q}_{r}\right)=(1030,2650)\right)$ than that for the CO setting $\left(\$ 50,412\right.$ with $\left.\left(q_{n}^{*}, q_{r}^{*}\right)=(1226,2392)\right)$.

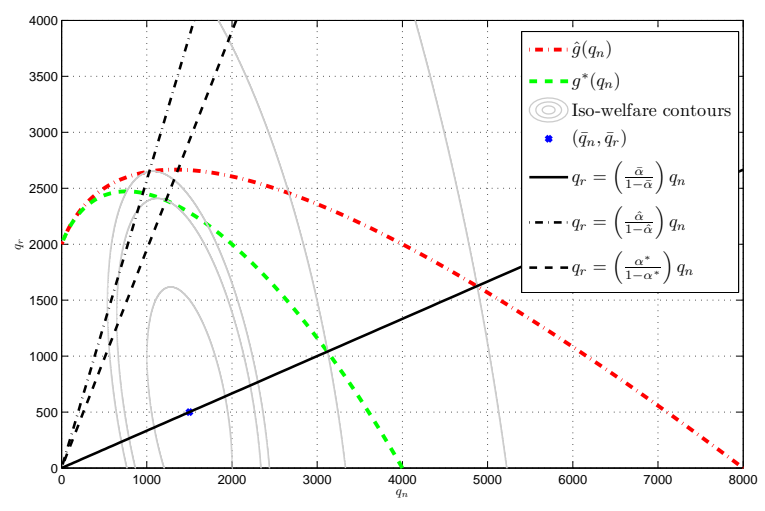

Figure 11: Iso-Welfare Contours and Loci of Optimal Solutions for the Lower Level for $K=0.04$

\subsection{Counterfactual Analysis}

Instead of a bi-level analysis, a policymaker may instead use the implied RPS target from solving a single-level QP in Eq. (1) as if the first-best outcome were possible. Since we have proven that the implied RPS target, $\bar{\alpha}$, is less than the optimal RPS target required by both the PC and CO settings, how much worse off would society be as a result of using the less stringent mandate? Such a counterfactual analysis is worth pondering for two reasons. First, the modelling effort necessary for a bi-level analysis may be too costly for a policy bureau, thereby necessitating the use of the implied RPS target from solving a QP as if one were operating under a CP setting. Second, the RPS targets that have been discussed in policy circles are considerably lower than the ones that would lead to deeper cuts in $\mathrm{CO}_{2}$ emissions. For example, a recent consulting report by E3 (Energy and Environmental Economics, 2014) finds that a $50 \%$ RPS target for California would lead to a $20 \%$ greater reduction in emissions than the baseline $33 \%$ target in the year 2030. However, the study also notes political (in 


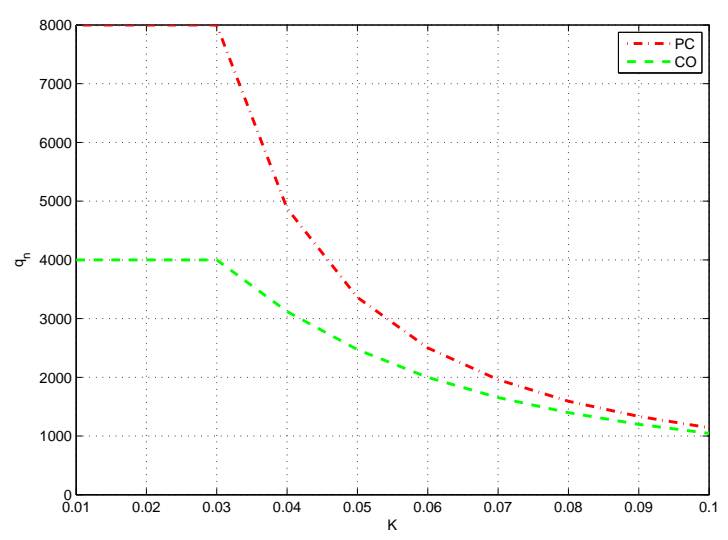

Figure 12: Optimal NRE Production using $\bar{\alpha}$

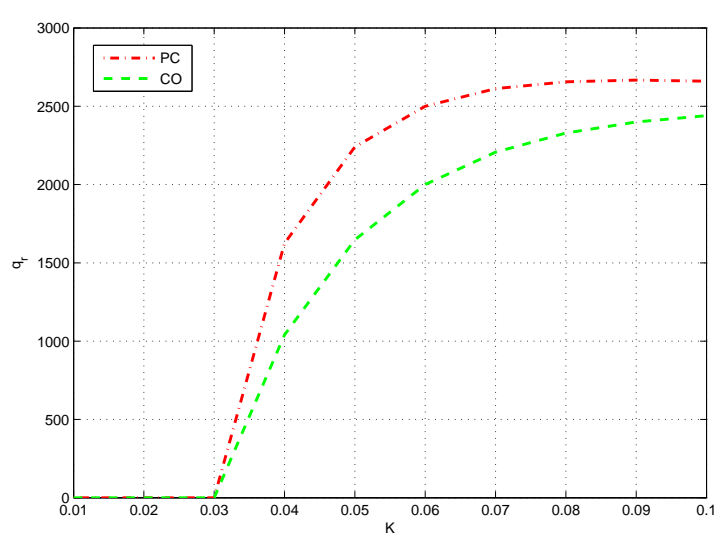

Figure 13: Optimal RE Production using $\bar{\alpha}$

the form of retail rate increases) and technical (due to overproduction of renewable energy) challenges associated with the higher RPS target. Thus, there may be valid reasons for policymakers to commit to more modest RPS targets. Consequently, we conduct a counterfactual analysis as in Nasiri and Zaccour (2010) here in order to investigate what may be the drawbacks of using more convenient RPS targets.

We run the lower-level MCP for the PC (Eqs. (14)-(16)) and the CO (Eqs. (15)-(16) and (28) ) while fixing the RPS target exogenously to its CP level, i.e., $\bar{\alpha}$. Comparing the production (Figs. 12 and 13), we find that the looser RPS target in the counterfactual analysis increases the NRE output and depresses the RE output (except for very high values of $K$ ). In fact, the NRE production increases even relative to the CP setting (Fig. 3) as the total consumption is not curbed in line with the damage costs of emissions.

The impact of fixing the RPS target to the CP proportion without curbing consumption to internalise the social cost of emissions is to lower the equilibrium electricity price (Fig. 14). In effect, adopting the RPS target from CP without either adjusting for the externality from emissions or offering the RE sector enough of a market share leads to an equilibrium electricity price that is too low to mitigate the effects of emissions optimally. Although the REC price under PC remains constant at $C_{r}-C_{n}$ (as long as $\bar{\alpha}>0$ ), the use of the CP RPS target under the CO setting lowers the REC price (Fig. 15). Consequently, the incentives for $\mathrm{RE}$ production in the $\mathrm{CO}$ setting are dampened and have less of an impact on emissions in the PC setting as consumption remains relatively high even as RE output increases.

Given the policymaker's lack of control over a deregulated electricity industry, its use of CP-like RPS targets may lead to large welfare losses precisely from increases in emissions. Indeed, while both consumers and producers in both PC and CO settings benefit from the use of counterfactual RPS targets (due to lower prices and higher output, respectively), the costs of externality are exacerbated (Fig. 16). Relative to the results in Fig. 7, the cost of damage 


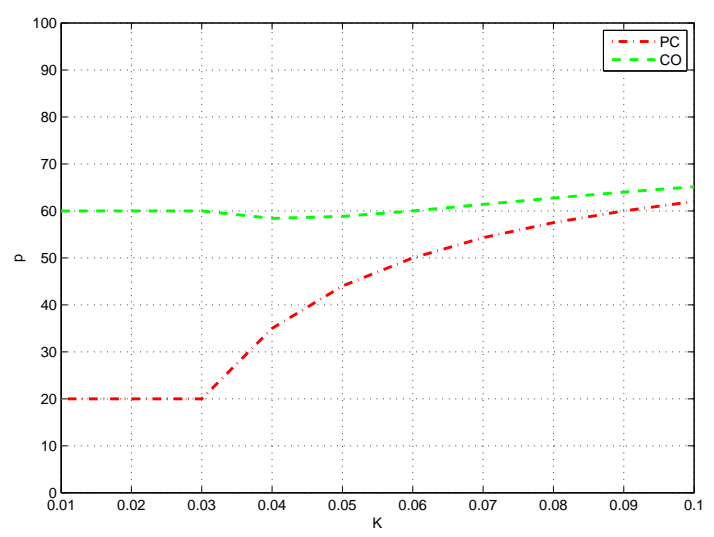

Figure 14: Equilibrium Electricity Price using $\bar{\alpha}$

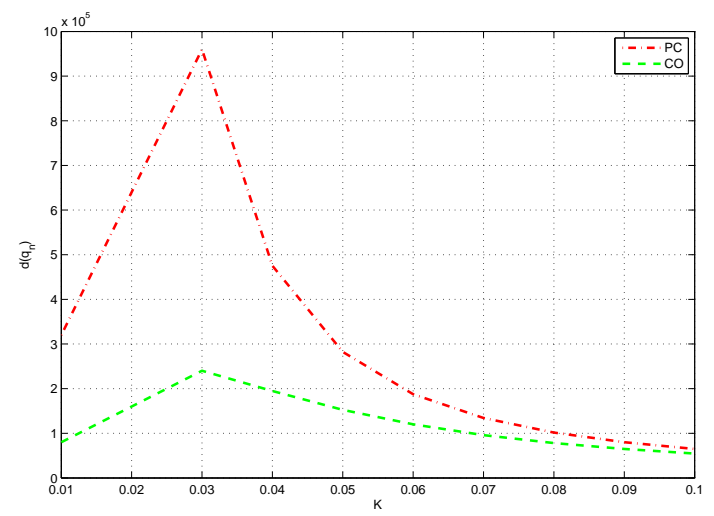

Figure 16: Cost of Damage from Emissions using $\bar{\alpha}$

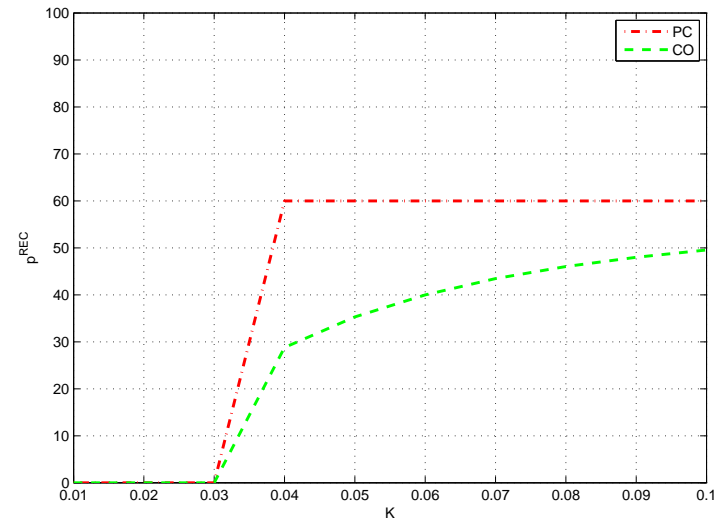

Figure 15: Equilibrium REC Price using $\bar{\alpha}$

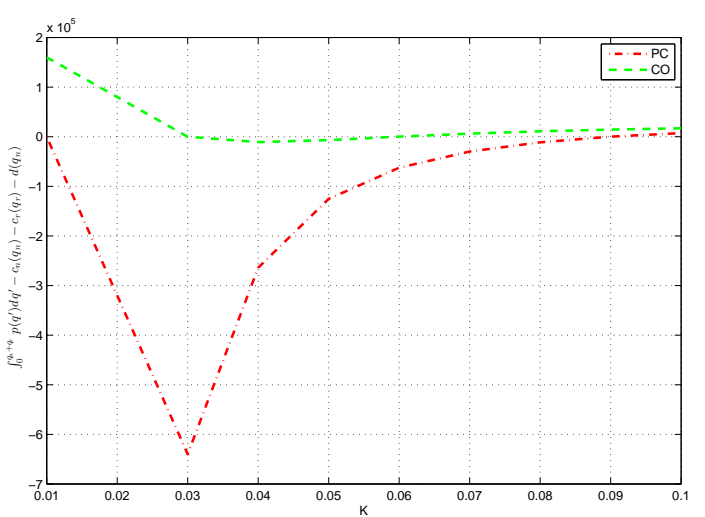

Figure 17: Maximised Social Welfare using $\bar{\alpha}$

from emissions is an order of magnitude higher under the counterfactual runs. Although higher values of $K$ reduce some of these discrepancies, significant differences remain in the cost of damage from emissions. Overall, this higher externality cost from the counterfactual runs leads to a substantial reduction in the social welfare (Fig. 17). Under the PC setting, there is a nearly $80 \%$ loss in welfare even for $K=0.1$, an impact that is somewhat lower for the $\mathrm{CO}$ setting at $50 \%$. Again, this mitigation of the loss in welfare under the CO setting may be attributed to the exercise of market power by the NRE sector, which curbs emissions. Nevertheless, the counterfactual runs illustrate that the consequences of policymakers' using easier-to-calculate RPS targets rather than those optimised for deregulated electricity industries will be reflected in higher emissions rather than direct losses to consumers or producers. 


\subsection{Discussion of Results}

By anticipating the profit-maximising production of lower-level NRE and RE sectors, the policymaker at the upper level is able only to adjust the RPS target rather than to intervene directly in the industry to assure a first-best outcome. Given this framework, we have following central findings in our paper:

- In comparison to a first-best benchmark setting with a central planner who controls all production, we find that RPS targets required in a decentralised industry are much higher. Nevertheless, the outcome in a centralised setting is socially optimal because the central planner is able to curb emissions by reducing consumption and NRE production. Only when the marginal cost of damage from emissions increases does it become necessary to introduce RE production. By contrast, in a decentralised industry, the policymaker has no control over consumption and production. Thus, its only leverage over producers is to mandate a more stringent RPS target, which leads to a decrease in emissions without reducing consumption extensively. Consequently, the nature of production and consumption outcomes is fundamentally different between centralised and decentralised settings.

- While the decentralised settings result in deeper reduction of emissions without curtailing consumption extensively, they, nevertheless, reduce social welfare relative to the centralised setting. This is because a central planner internalises the cost of damage from emissions and effectively treats it as a Pigouvian tax. Correspondingly, the marginal benefit of consumption is precisely aligned with the marginal cost of production, inclusive of the externality. Since the decentralised settings use the RPS to control emissions, they result in "too much" RE production because the REC is an economic distortion, i.e., a subsidy for the RE sector and a tax on the NRE sector.

- Intriguingly, the effect of this distortion is mitigated when the NRE sector is able to exercise power, viz., by withholding production in order to raise the electricity price and to lower the REC price. We, therefore, obtain the surprising result that social welfare is actually higher when the NRE sector behaves $\grave{a} l a$ Cournot.

\section{Conclusions}

In order to mitigate climate change, transitions towards more sustainable energy systems have been proposed by the EU and many American states. For the electricity industry, the foreseen reductions in $\mathrm{CO}_{2}$ emissions require even more drastic measures as many sectors of 
the economy, e.g., transportation, will rely on electrification to comply with targets. Complicating the situation for policymakers is the fact that electricity industries were deregulated in most OECD countries over the past thirty years in order to improve economic efficiency by giving producers the price signals to commercialise new technologies. Thus, somewhat paradoxically, policymakers have ceded more control over electricity industries while at the same time requiring them to shift more towards renewable sources. In order to manage this dilemma, the market-based renewable portfolio standard mechanism has been implemented in many jurisdictions to incentivise higher production from the renewable energy sector.

In this paper, we take the perspective of a welfare-maximising policymaker who sets the optimal RPS target but has no direct control over the production and consumption decisions of a deregulated electricity industry. This separation is formalised via a bi-level model, and unlike most other complementarity-based analyses of RPS, we make the target level endogenous. We find that in a benchmark setting with a centralised electricity industry, the costs of damage from emissions are internalised, thereby resulting in a substantial curb in consumption and concomitant decrease in NRE production. Once the social cost of NRE output equals the cost of RE production, consumption is no longer reduced, and NRE production is substituted with RE, which means that the first-best solution is effectively to implement a Pigouvian tax on NRE production. This implied RPS target turns out to be lower than that in the $\mathrm{PC}$ and $\mathrm{CO}$ settings with deregulated electricity industries.

The seemingly counterintuitive ranking of RPS targets may be explained by the fact that a policymaker in a deregulated industry no longer has control over production and consumption decisions. As a result, it cannot internalise the cost of damage from emissions to allocate resources efficiently. However, it can impose more stringent RPS targets, which lead to more RE production even as consumption remains higher than in the $\mathrm{CP}$ setting. Thus, the policymaker mitigates the impact of "too much" consumption by inducing substantially more RE production. Relative to the socially optimal (but politically unrealistic) outcome of the CP setting, the policymaker introduces an economic inefficiency to counter the effects of an externality. For this reason, although the cost of damage from emissions is lower under the PC and CO settings, the maximised social welfare in the CP setting is actually higher. Moreover, this distortion is reduced under the CO setting as the NRE sector's exercise of market power subverts the RE sector's position.

Given the difficulties of calibrating a bi-level model to capture the details of a deregulated electricity industry and the political as well as technical challenges of mandating higher RPS targets, it may seem reasonable for policymakers to use the easier-to-calculate implied RPS targets from the CP setting. However, because these lower RPS targets maximise social welfare only by curbing total consumption, which is another political difficulty in a deregulated 
industry, their use in reality may substantially worsen outcomes. Indeed, our counterfactual analysis indicates that setting RPS targets while not accounting for the deregulated nature of electricity industries may lead to a significant increase in the cost of damage from emissions and, thus, substantial welfare losses. Somewhat surprisingly, the welfare losses are actually higher the less the potential for exercise of market power. Therefore, policymakers who are contemplating RPS targets should craft them carefully to account for the incentives of producers.

For future work, we envisage constructing a model that considers the realities of power systems such as transmission constraints, nodal prices, and uncertainty in RE output as in Baringo and Conejo (2013) and Maurovich-Horvat et al. (2015). The interaction of RPS targets with smart-grid technologies such as electric vehicles and storage would also merit investigation. Finally, allowing generation capacity expansion over time in a multi-period model would enable a long-term analysis of RPS.

\section{Acknowledgements}

We are grateful to Ekaterina V. Moiseeva and Ahmad S. Siddiqui for feedback on an earlier version of the paper. Comments from attendees of the 2014 IAEE International Conference, the 2014 INFORMS Annual Meeting, and the OR56 Conference as well as seminars at NTNU, GERAD, and Imperial College have helped to improve this paper. Input from three anonymous referees has also bolstered the work's exposition and positioning. Siddiqui is grateful to the RISKY-RES project (no. 228811) funded by the Research Council of Norway for support. Any remaining errors are the authors' own.

\section{References}

Adelaja, S.J., Y.G. Hailu (2008). Effects of renewable portfolio standards and other state policies on wind industry development in the U.S. Mimeo, Michigan State University, East Lansing, MI.

Allaz, B., J.-L. Vila (1993). Cournot competition, forward markets and efficiency. J. Econ. Theory 59:1-16.

Amundsen, E.S., L. Bergman (2012). Green certificates and market power on the Nordic power market. The Energy J. 33(2):101-117.

Amundsen, E.S., J.B. Mortensen (2001). The Danish green certificate system: some simple analytical results. Energy Econ. 23:489-509.

Baringo, L., A.J. Conejo (2013). Transmission and wind power investment. IEEE Trans. on Power Syst. 27(2):885-893. 
Bird, L.A., E. Lokey (2007). Interaction of compliance and voluntary renewable energy market. Tech. Rep. NREL/TP-670-42096, National Renewable Energy Laboratory, Golden, $\mathrm{CO}$.

Boomsma, T.K., N. Meade, S.-E. Fleten (2012). Renewable energy investments under different support schemes: A real options approach. Eur. J. of Oper. Res. 220(1):225-237.

Chen, Y., A.L. Liu, B.F. Hobbs (2011). Economic and emissions implications of load-based, source-based, and first-seller emissions trading programs under California AB32. Oper. Res. 59(3):696-712.

Chen, Y., L. Wang (2013). Renewable portfolio standards in the presence of green consumers and emissions trading. Networks and Spatial Econ. 13:149-181.

$\begin{array}{ccccccc}\text { Energy } & \text { and } & \text { Environmental } & \text { Economics } & \text { Investigat- } \\ \text { ing } & \text { a } & \text { Higher } & \text { Renewables } & \text { Portfolio } & \text { Standard in } & \text { California. }\end{array}$
https://ethree.com/documents/E3_Final_RPS_Report_2014_01_06_with_appendices.pdf, San Francisco, CA.

European Commission (2009). Directive 2009/28/EC of the European Parliament and of the Council. http://http://eur-lex.europa.eu/legal-content/EN/TXT/?uri=CELEX:32009L0028, Brussels, Belgium.

Fischer, C. (2010). Renewable portfolio standards: when do they lower energy prices? The Energy J. 31(1):101-120.

Gabriel, S.A., A.J. Conejo, J.D. Fuller, B.F. Hobbs, C. Ruiz (2012). Complementarity Modeling in Energy Markets. Springer, Germany.

Hibiki, A., Y. Kurakawa (2013). Which is a better second best policy, the feed-in tariff scheme or the renewable portfolio standard scheme? RIETI Discussion Paper Series 13-J070, Research Institute of Economy, Trade \& Industry, Tokyo, Japan (in Japanese).

Holt, E., R. Wiser (2007).The treatment of renewable energy certificates, emissions allowances, and green power programs in state renewable portfolio standards. Tech. Rep. LBNL-62574, Ernest Orlando Lawrence Berkeley National Laboratory, Berkeley, CA.

Kneifel, J.D. (2008). Essays in Renewable Energy and Emissions Trading. Ph.D. Dissertation, Department of Economics, University of Florida, Gainesville, Florida.

Maurovich-Horvat, L., T.K. Boomsma, A.S. Siddiqui (2015). Transmission and wind investment in a deregulated electricity industry. IEEE Trans. on Power Syst. 30(3):1633-1643. 
Menz, F.C., S. Vachon (2006). The effectiveness of different policy regimes for promoting wind power: experience from the states. Energy Policy 34:1786-1796.

Murphy, F., E. Rosenthal (2006). Allocating the added value of energy policies. The Energy J. $27(2): 143-156$.

Murphy, F., Y. Smeers (2010). On the impact of forward markets on investments in oligopolistic markets with reference to electricity. Oper. Res. 58(3):515-528.

Nasiri, F., G. Zaccour (2010). Renewable portfolio standard policy: a game-theoretic analysis. INFOR 48(4):251-260.

Sauma, E.E., S.S. Oren (2006). Proactive planning and valuation of transmission investments in restructured electricity markets. J. of Reg. Econ. 30:261-290.

Tanaka, M., Y. Chen (2013). Market power in renewable portfolio standards. Energy Econ. 39:187-196.

Wiser, R., G. Barbose (2008). Renewables portfolio standards in the United States: A status report with data through 2007. Tech. Rep., Ernest Orlando Lawrence Berkeley National Laboratory, Berkeley, CA.

Yin, H., N. Powers (2010). Do State Renewable Portfolio Standards Promote In-state Renewable Generation? Energy Policy 38(2):1140-1149.

Zhou, Y., L. Wang, J.D. McCalley (2011). Designing effective and efficient incentive policies for renewable energy in generation expansion planning. Applied Energy 88:2201-2209.

\section{Appendix: Proofs of Propositions}

Proof of Proposition 1 The results follow from differentiating the CP solutions in Eqs. (44)-(8) with respect to $K$.

Proof of Proposition 2 The results follow from differentiating the expressions in Eqs. (17) -(20) with respect to $\alpha$.

Proof of Proposition 3 Taking the total differential of $\hat{\mathcal{Q}}(\alpha, K)$ and evaluating it at $\alpha=$ $\hat{\alpha}$, we have $\frac{d \hat{\alpha}}{d K}=-\left.\frac{\frac{\partial \hat{\mathcal{Q}}}{\partial K}}{\frac{\partial \hat{\mathcal{Q}}}{\partial \alpha}}\right|_{\alpha=\hat{\alpha}}$. We would like to show that this expression is positive. The proof follows because first, its numerator, $\frac{\partial \hat{\mathcal{Q}}}{\partial K}$, is equal to $(1-\hat{\alpha})\left[\left(A-C_{n}\right)+\left(C_{r}-C_{n}\right)(1-2 \hat{\alpha})\right]$, which may be re-written as $(1-\hat{\alpha})\left\{\left[A-\left(\hat{\alpha} C_{r}+(1-\hat{\alpha}) C_{n}\right)\right]+\left(C_{r}-C_{n}\right)(1-\hat{\alpha})\right\}$. This expression is positive because both terms inside the curly brackets are positive. From the geometry of $\hat{\mathcal{Q}}(\alpha, K)$, we also know that the denominator in the expression for $\frac{d \hat{\alpha}}{d K}$ is negative. Specifically, $\left.\frac{\partial \hat{\mathcal{Q}}}{\partial \alpha}\right|_{\alpha=0}=-3 K\left(C_{r}-C_{n}\right)-K\left(A-C_{n}\right)<0$ and $\left.\frac{\partial \hat{\mathcal{Q}}}{\partial \alpha}\right|_{\alpha=1}=-K\left(A-C_{r}\right)<0$. 
In other words, $\frac{\partial \hat{\mathcal{Q}}}{\partial \alpha}<0 \forall \alpha \in[0,1]$ because the derivative of the parabola $\hat{\mathcal{Q}}(\alpha, K)$ changes sign only once. Hence, the overall expression for $\frac{d \hat{\alpha}}{d K}$ is positive.

Proof of Proposition 4 Suppose that it is the contrary, i.e., assume $\hat{\alpha} \leq \bar{\alpha}$. Fixing $K$, we define $\xi(\alpha) \equiv \hat{\mathcal{Q}}(\alpha, K)-\overline{\mathcal{Q}}(\alpha, K)$. Using the expressions for the characteristic functions, we obtain $\xi(\alpha)=2 K\left(C_{r}-C_{n}\right)(1-\alpha)^{2}$, which is also a convex quadratic with multiple roots at $\alpha=1$. Thus, $\xi(\alpha)>0 \forall \alpha \in(0,1)$. Furthermore, $\xi^{\prime}(\alpha)=4 K\left(C_{r}-C_{n}\right)(\alpha-1)<0 \forall \alpha \in$ $(0,1)$. Putting these attributes together, we have $0 \equiv \hat{\mathcal{Q}}(\hat{\alpha}, K) \geq \hat{\mathcal{Q}}(\bar{\alpha}, K)>\overline{\mathcal{Q}}(\bar{\alpha}, K) \equiv 0$. The first inequality follows from the assumption that $\hat{\alpha} \leq \bar{\alpha}$ and the fact that $\hat{\mathcal{Q}}(\alpha, K)$ is monotonically decreasing. Next, the second inequality stems from the fact that $\xi(\alpha)$ is strictly positive for $\alpha \in(0,1)$. Hence, we run into a contradiction and conclude that $\hat{\alpha}>$ $\bar{\alpha}$.

Proof of Proposition 5 The sign of $\hat{\mathcal{Q}}(\alpha, K)$ corresponds to the slope of the objective function in Eq. (21). We have $\hat{\mathcal{Q}}(1, K)=-\left(C_{r}-C_{n}\right) Z<0,\left.\frac{\partial \hat{\mathcal{Q}}}{\partial \alpha}\right|_{\alpha=1}=-\left(A-C_{r}\right) K<0$, and $\hat{\mathcal{Q}}(0, K)=Y(K)>0$. Therefore, the quadratic function $\hat{\mathcal{Q}}(\alpha, K)$ crosses the horizontal axis from positive to negative exactly once for $0<\alpha<1$, which means that the policymaker's problem in Eq. (21) has a unique maximum at $\hat{\alpha} \in(0,1)$ that satisfies $\hat{\mathcal{Q}}(\hat{\alpha}, K)=0$.

Proof of Proposition 6 The results follow from a combination of Propositions 2 and 3 ;

(i) $\frac{d \hat{q}_{n}(K)}{d K}=\frac{\partial \hat{q}_{n}}{\partial \alpha} \frac{\partial \alpha}{\partial K}<0$

(ii) $\frac{d \hat{q}_{r}(K)}{d K}=\frac{\partial \hat{q}_{r}}{\partial \alpha} \frac{\partial \alpha}{\partial K}$

This is ambiguous in sign.

(iii) $\frac{d \hat{q}(K)}{d K}=\frac{\partial \hat{q}}{\partial \alpha} \frac{\partial \alpha}{\partial K}<0$

(iv) $\frac{d \hat{p}^{R E C}(K)}{d K}=0$

(v) $\frac{d \hat{p}(K)}{d K}=\frac{\partial \hat{p}}{\partial \alpha} \frac{\partial \alpha}{\partial K}>0$

Proof of Proposition 7 Suppose not, i.e., assume that $\hat{p}(\alpha) \geq p^{*}(\alpha)$. Using the interior solutions for $\hat{p}(\alpha)$ and $p^{*}(\alpha)$ in Eqs. (201) and (32), respectively, we obtain the following: $\alpha C_{r}+(1-\alpha) C_{n} \geq \frac{A(1-\alpha)^{2}+\alpha C_{r}+(1-\alpha) C_{n}}{F(\alpha)} \Rightarrow\left[\alpha C_{r}+(1-\alpha) C_{n}\right](F(\alpha)-1)-A(1-\alpha)^{2} \geq 0$ $\Rightarrow A-\left[\alpha C_{r}+(1-\alpha) C_{n}\right] \leq 0$

Since the last line contradicts the assumption that $A-\left(\alpha C_{r}+(1-\alpha) C_{n}\right)>0$, it must be the case that $\hat{p}(\alpha)<p^{*}(\alpha)$. 
Proof of Proposition 8 Suppose not, i.e., assume that $\hat{p}^{R E C}(\alpha) \leq p^{R E C^{*}}(\alpha)$. Using the interior solutions for $\hat{p}^{R E C}(\alpha)$ and $p^{R E C^{*}}(\alpha)$ in Eqs. (19) and (31), respectively, we obtain the following:

$\left(C_{r}-C_{n}\right) F(\alpha) \leq\left(C_{r}-C_{n}\right)-(1-\alpha)\left(A-C_{r}\right) \Rightarrow\left(C_{r}-C_{n}\right)(F(\alpha)-1)+(1-\alpha)\left(A-C_{r}\right) \leq$ $0 \Rightarrow A-\left(\alpha C_{r}+(1-\alpha) C_{n}\right) \leq 0$

Since the last line contradicts the assumption that $A-\left(\alpha C_{r}+(1-\alpha) C_{n}\right)>0$, it must be the case that $\hat{p}^{R E C}(\alpha)>p^{R E C^{*}}(\alpha)$.

Proof of Proposition 9 From Eqs. (17), (18), (29), and (30), $q_{n}^{*}(\alpha)=\frac{\hat{q}_{n}(\alpha)}{F(\alpha)}$ and $q_{r}^{*}(\alpha)=$ $\frac{\hat{q}_{r}(\alpha)}{F(\alpha)}$ hold. Noting that $F(\alpha)>1$ for $\alpha \in(0,1)$, we have $q_{n}^{*}(\alpha)<\hat{q}_{n}(\alpha)$ and $q_{r}^{*}(\alpha)<$ $\hat{q}_{r}(\alpha)$.

Proof of Proposition 10 The sign of $\mathcal{Q}^{*}(\alpha, K)$ corresponds to the slope of the objective function in Eq. (33). We show that $\mathcal{Q}^{*}(\alpha, K)$ crosses the horizontal axis from positive to negative exactly once for $0<\alpha<1$, which means that the policymaker's problem in Eq. (33) has a unique maximum at $\alpha^{*} \in(0,1)$ that satisfies $\mathcal{Q}^{*}\left(\alpha^{*}, K\right)=0$. We first note $\mathcal{Q}^{*}(1, K)=\overline{\mathcal{Q}}(1, K)=-\left(C_{r}-C_{n}\right) Z<0$ and $\left.\frac{\partial \mathcal{Q}^{*}}{\partial \alpha}\right|_{\alpha=1}=\left.\frac{\partial \overline{\mathcal{Q}}}{\partial \alpha}\right|_{\alpha=1}=-\left(A-C_{r}\right) K<0$, which implies that $\mathcal{Q}^{*}(\alpha, K)$ and $\overline{\mathcal{Q}}(\alpha, K)$ are tangent at $\alpha=1$. Note also that $\mathcal{Q}^{*}(0, K)>0$ since $\mathcal{Q}^{*}(0, K)=2\left(A-C_{r}\right) Z+2(K-2 Z)\left(C_{r}-C_{n}\right)>\frac{2 Z^{2}}{K}\left(C_{r}-C_{n}\right)+2(K-2 Z)\left(C_{r}-C_{n}\right)=$ $\frac{2(K-Z)^{2}}{K}\left(C_{r}-C_{n}\right)>0$. The first inequality is from the interior assumption $\overline{\mathcal{Q}}(0, K)>0$. There are three cases to consider:

(i) If $K-2 Z>0$, then the coefficient of $\alpha^{3}$ in the cubic function $\mathcal{Q}^{*}(\alpha, K)$ is positive, which implies that the second derivative changes from negative to positive as $\alpha$ increases. Noting that $\mathcal{Q}^{*}(1, K)<0,\left.\frac{\partial \mathcal{Q}^{*}}{\partial \alpha}\right|_{\alpha=1}<0$, and $\mathcal{Q}^{*}(0, K)>0, \mathcal{Q}^{*}(\alpha, K)$ crosses the horizontal axis from positive to negative only once for $0<\alpha<1$.

(ii) If $K-2 Z=0$, then $\mathcal{Q}^{*}(\alpha, K)$ becomes a quadratic function, and it also intersects the horizontal axis from positive to negative only once for $0<\alpha<1$.

(iii) If $K-2 Z<0$, then the coefficient of $\alpha^{3}$ in the cubic function $\mathcal{Q}^{*}(\alpha, K)$ is negative, which implies that the second derivative changes from positive to negative as $\alpha$ increases. When $\left.\frac{\partial^{2} \mathcal{Q}^{*}}{\partial \alpha^{2}}\right|_{\alpha=1} \geq 0, \mathcal{Q}^{*}(\alpha, K)$ is monotonically decreasing above $\overline{\mathcal{Q}}(\alpha, K)$ for $0<\alpha<1$, and both are are tangent at $\alpha=1$. Thus, $\mathcal{Q}^{*}(\alpha, K)$ intersects the horizontal axis from positive to negative only once for $0<\alpha<1$. When $\left.\frac{\partial^{2} \mathcal{Q}^{*}}{\partial \alpha^{2}}\right|_{\alpha=1}<0$, $\mathcal{Q}^{*}(\alpha, K)$ has an intersection with $\overline{\mathcal{Q}}(\alpha, K)$ at $\alpha=1-\frac{\left(C_{r}-C_{n}\right)(2 K-3 Z)}{\left(A-C_{r}\right)(K-2 Z)}<1$ such that $Q^{*}\left(\alpha^{\prime}\right)=\bar{Q}\left(\alpha^{\prime}\right)=\frac{2\left(C_{r}-C_{n}\right)(K-Z)^{2}}{(K-2 Z)}<0$. Consequently, $\mathcal{Q}^{*}(\alpha, K)$ and $\overline{\mathcal{Q}}(\alpha, K)$ are tangent at $\alpha=1$. This implies that $\mathcal{Q}^{*}(\alpha, K)$ crosses $\overline{\mathcal{Q}}(\alpha, K)$ only once from above. Hence, $\mathcal{Q}^{*}(\alpha, K)$ intersects the horizontal axis from positive to negative only once at $0<\alpha<\dot{\alpha}<1$. 
Proof of Proposition 11 Taking the total differential of $\mathcal{Q}^{*}(\alpha, K)$ and evaluating it at $\alpha=\alpha^{*}$, we have $\frac{d \alpha^{*}}{d K}=-\left.\frac{\frac{\partial \mathcal{Q}^{*}}{\partial \mathcal{Q}^{*}}}{\frac{\partial \alpha}{\partial \alpha}}\right|_{\alpha=\alpha^{*}}$. We would like to show that this expression is positive. The proof follows because first, its numerator, $\frac{\partial \mathcal{Q}^{*}}{\partial K}$, is equal to:

$$
\left(1-\alpha^{*}\right)\left\{\left(C_{r}-C_{n}\right)\left(1-\alpha^{*}\right) F\left(\alpha^{*}\right)+\left[A-\left(\alpha^{*} C_{r}+\left(1-\alpha^{*}\right) C_{n}\right)\right]\left(F\left(\alpha^{*}\right)-2\left(1-\alpha^{*}\right)^{3}\right)\right\}
$$

This expression is positive because the first term inside the curly brackets is positive, and the second one may be re-expressed as $\left[A-\left(\alpha^{*} C_{r}+\left(1-\alpha^{*}\right) C_{n}\right)\right]\left(\left(1-\alpha^{*}\right)^{2}\left(2 \alpha^{*}-1\right)+1\right)$. Since this is positive $\forall \alpha^{*} \in(0,1)$, we prove that $\frac{\partial \mathcal{Q}^{*}}{\partial K}>0$. Next, from Proposition 10 we know that $\alpha^{*}$ constitutes a globally optimal solution to the policymaker's problem under the CO setting. In other words, the second-order condition must be satisfied at $\alpha^{*}$, thereby implying that the second derivative of the objective function in Eq. (33) is negative. Consequently, we have $\left.\frac{\partial \mathcal{Q}^{*}}{\partial \alpha}\right|_{\alpha=\alpha^{*}}<0$. Hence, we prove that $\frac{d \alpha^{*}}{d K}>0$.

Proof of Proposition 12 Eliminating $\alpha$ from parametric Eqs. (17) and (18), we derive an implicit function $\hat{G}\left(q_{n}, q_{r}\right)=0$ for PC:

$$
\hat{G}\left(q_{n}, q_{r}\right)=Z\left(q_{n}+q_{r}\right)^{2}-\left(A-C_{n}\right) q_{n}-\left(A-C_{r}\right) q_{r}=0
$$

which exhibits a conic curve. Furthermore, the locus within the range $0<\alpha<1$ that satisfies Eqs. (14)-(16) for the lower level of PC can be derived explicitly as follows:

$$
q_{r}=\hat{g}\left(q_{n}\right)=\frac{A-C_{r}-2 Z q_{n}+\sqrt{\left(A-C_{r}\right)^{2}+4 Z\left(C_{r}-C_{n}\right) q_{n}}}{2 Z}
$$

$\hat{g}\left(q_{n}\right)$ is strictly concave as simple calculation yields $\hat{g}^{\prime \prime}\left(q_{n}\right)<0$. Similarly, from Eqs. (29) and (30), we can derive an implicit function $G^{*}\left(q_{n}, q_{r}\right)=0$ for CO:

$$
G^{*}\left(q_{n}, q_{r}\right)=Z\left(q_{n}+q_{r}\right)^{2}+Z q_{n}^{2}-\left(A-C_{n}\right) q_{n}-\left(A-C_{r}\right) q_{r}=0
$$

which also shows a conic curve. Moreover, the locus within the range $0<\alpha<1$ that satisfies Eqs. (15)-(16) and (28) for CO can be expressed explicitly as follows:

$$
q_{r}=g^{*}\left(q_{n}\right)=\frac{A-C_{r}-2 Z q_{n}+\sqrt{\left(A-C_{r}\right)^{2}+4 Z\left(C_{r}-C_{n}\right) q_{n}-4 Z^{2} q_{n}^{2}}}{2 Z}
$$

$g^{*}\left(q_{n}\right)$ is strictly concave as simple calculation yields $g^{* \prime \prime}\left(q_{n}\right)<0$. From Eqs. (A-2) and (A-4), $\hat{g}\left(q_{n}\right)>g^{*}\left(q_{n}\right)$ holds, which implies that the locus $\hat{g}\left(q_{n}\right)$ for PC is above the locus $g^{*}\left(q_{n}\right)$ for CO. We then compare the loci for PC/CO and the interior optimum $\left(\bar{q}_{n}, \bar{q}_{r}\right)$ for 
CP. Simple calculation yields:

$$
\begin{gathered}
\hat{g}\left(\bar{q}_{n}\right)-\bar{q}_{r}=\frac{-\left(A-C_{r}\right)+\sqrt{\left(A-C_{r}\right)^{2}+\frac{4 Z\left(C_{r}-C_{n}\right)^{2}}{K}}}{2 Z}>0 \\
g^{*}\left(\bar{q}_{n}\right)-\bar{q}_{r}=\frac{-\left(A-C_{r}\right)+\sqrt{\left(A-C_{r}\right)^{2}+\frac{4 Z(K-Z)\left(C_{r}-C_{n}\right)^{2}}{K^{2}}}}{2 Z}>0
\end{gathered}
$$

Hence, the loci for PC/CO are above the optimum $\left(\bar{q}_{n}, \bar{q}_{r}\right)$ for CP. Note that an outer isowelfare contour corresponds to lower social welfare from the strict concavity of the CP's objective function in Eq. (1). Thus, the maximised social welfare for CP is greater than that for $\mathrm{PC} / \mathrm{CO}$. Furthermore, the iso-welfare contour that is tangent to the locus for PC should be located outside that for $\mathrm{CO}$. This implies that the maximised social welfare for $\mathrm{CO}$ is greater than that for PC.

Proof of Proposition 13 We first derive the slopes of the loci for PC and CO. Taking the total differentials of Eqs. (A-1) and (A-3) and evaluating them at $\left(\hat{q}_{n}(\alpha), \hat{q}_{r}(\alpha)\right)$ and $\left(q_{n}^{*}(\alpha), q_{r}^{*}(\alpha)\right)$, respectively, we have

$$
\begin{aligned}
\hat{S}(\alpha)=\frac{d \hat{q}_{r}(\alpha)}{d \hat{q}_{n}(\alpha)}=-\left.\frac{\frac{\partial \hat{G}}{\partial q_{n}}}{\frac{\partial \hat{G}}{\partial q_{r}}}\right|_{\left(q_{n}, q_{r}\right)=\left(\hat{q}_{n}(\alpha), \hat{q}_{r}(\alpha)\right)}=-\frac{A-C_{n}-2 \alpha\left(C_{r}-C_{n}\right)}{A+C_{r}-2\left[\alpha C_{r}+(1-\alpha) C_{n}\right]} \\
S^{*}(\alpha)=\frac{d q_{r}^{*}(\alpha)}{d q_{n}^{*}(\alpha)}=-\left.\frac{\frac{\partial G^{*}}{\partial q_{n}}}{\frac{\partial G^{*}}{\partial q_{r}}}\right|_{\left(q_{n}, q_{r}\right)=\left(q_{n}^{*}(\alpha), q_{r}^{*}(\alpha)\right)} \\
=-\frac{\left(\alpha^{2}-2\right) A+2 \alpha(\alpha-2) C_{r}+(F(\alpha)-2 \alpha) C_{n}}{\alpha(\alpha-2)\left(A-C_{r}\right)+2\left[\alpha C_{r}+(1-\alpha) C_{n}-C_{r}\right]}
\end{aligned}
$$

The slope of the iso-welfare contour can be also obtained from Eq. (1) as follows:

$$
\bar{S}\left(q_{n}, q_{r}\right)=\frac{d q_{r}}{d q_{n}}=-1-\frac{C_{r}-C_{n}-K q_{n}}{A-C_{r}-Z\left(q_{n}+q_{r}\right)}
$$

We first prove $\bar{\alpha}<\alpha^{*}$ by comparing the slopes, $S^{*}$ and $\bar{S}$, at the locus point $\left(q_{n}^{*}(\alpha), q_{r}^{*}(\alpha)\right)$ of $\mathrm{CO}$ when evaluated at $\alpha=\bar{\alpha}$. In terms of Fig. 11, we draw a line from the origin, passing through the point $\left(\bar{q}_{n}, \bar{q}_{r}\right)$ corresponding to $\alpha=\bar{\alpha}$, and examine the intersection of this line and the locus for $\mathrm{CO}$. We calculate the difference in the slopes as follows:

$$
S^{*}(\bar{\alpha})-\bar{S}\left(q_{n}^{*}(\bar{\alpha}), q_{r}^{*}(\bar{\alpha})\right)=\frac{2\left(C_{r}-C_{n}\right)(K-Z)\left[K\left(A-C_{r}\right)^{2}+Z\left(C_{r}-C_{n}\right)^{2}\right]}{\left(A-C_{r}\right)\left[K^{2}\left(A-C_{r}\right)^{2}+Z(2 K-Z)\left(C_{r}-C_{n}\right)^{2}\right]}>0
$$


Thus, $S^{*}(\bar{\alpha})>\bar{S}\left(q_{n}^{*}(\bar{\alpha}), q_{r}^{*}(\bar{\alpha})\right)$ holds, i.e., the slope of the CO locus takes a greater value than that of the iso-welfare contour at $\left(q_{n}^{*}(\bar{\alpha}), q_{r}^{*}(\bar{\alpha})\right)$. This means that we can find an inner iso-welfare contour that is tangent to the locus for $\mathrm{CO}$ at a point $\left(q_{n}^{*}\left(\alpha^{*}\right), q_{r}^{*}\left(\alpha^{*}\right)\right)$ to the left of $\left(q_{n}^{*}(\bar{\alpha}), q_{r}^{*}(\bar{\alpha})\right)$. Otherwise, the slopes would diverge further and not lead to a tangency between the iso-welfare contour and the CO locus. Therefore, $\bar{\alpha}<\alpha^{*}$ holds true, noting that $\frac{q_{r}}{q_{n}}=\frac{\alpha}{1-\alpha}$ and that $\bar{\alpha}$ and $\alpha^{*}$ are unique solutions for $0<\alpha<1$.

Next, we prove $\alpha^{*}<\hat{\alpha}$ by comparing the slopes at the locus points of $\mathrm{CO}$ and $\mathrm{PC}$ for some given $\alpha$. In other words, we draw a line from the origin for some given $\alpha$ and examine the intersections of this line and the loci for $\mathrm{CO}$ and PC. For a given $\alpha$, we calculate the difference between the slopes of the loci as follows:

$$
\begin{aligned}
& \hat{S}(\alpha)-S^{*}(\alpha) \\
= & \frac{2(1-\alpha)\left[A-\alpha C_{r}-(1-\alpha) C_{n}\right]^{2}}{\left\{A+C_{r}-2\left[\alpha C_{r}+(1-\alpha) C_{n}\right]\right\}\left\{\alpha(2-\alpha)\left(A-C_{r}\right)+2\left[C_{r}-\alpha C_{r}-(1-\alpha) C_{n}\right]\right\}} \\
> & 0
\end{aligned}
$$

This implies that the slope of the PC locus takes a greater value than that of the CO locus at any arbitrary $\alpha$. For a given $\alpha$, the difference of the slopes of the iso-welfare contours at the locus points of $\mathrm{CO}$ and $\mathrm{PC}$ can also be derived as follows:

$$
\begin{aligned}
\bar{S}\left(q_{n}^{*}(\alpha), q_{r}^{*}(\alpha)\right) & -\bar{S}\left(\hat{q}_{n}(\alpha), \hat{q}_{r}(\alpha)\right) \\
& =\frac{\left[A-\alpha C_{r}-(1-\alpha) C_{n}\right]\left[Z\left(C_{r}-C_{n}\right)-(1-\alpha) K\left(A-C_{r}\right)\right]}{Z\left(C_{r}-C_{n}\right)\left[\left(C_{r}-C_{n}\right)-(1-\alpha)\left(A-C_{r}\right)\right]}
\end{aligned}
$$

The expression in Eq. ( $(\mathrm{A-12})$ is greater than zero for any $\alpha>\bar{\alpha}=1-\frac{Z\left(C_{r}-C_{n}\right)}{K\left(A-C_{r}\right)}>0$, which is the relevant case for our analysis. Now, consider the optimal RPS target $\alpha^{*}$ for CO. From the tangency condition of the CO locus and iso-welfare contour, $S^{*}\left(\alpha^{*}\right)=\bar{S}\left(q_{n}^{*}\left(\alpha^{*}\right), q_{r}^{*}\left(\alpha^{*}\right)\right)$. On the other hand, regarding the PC locus, we have $\hat{S}\left(\alpha^{*}\right)>S^{*}\left(\alpha^{*}\right)$ and $\bar{S}\left(q_{n}^{*}\left(\alpha^{*}\right), q_{r}^{*}\left(\alpha^{*}\right)\right)>$ $\bar{S}\left(\hat{q}_{n}\left(\alpha^{*}\right), \hat{q}_{r}\left(\alpha^{*}\right)\right)$ from Eqs. (A-11) and (A-12), respectively (see Fig. 11). Consequently, we have the relation $\hat{S}\left(\alpha^{*}\right)>S^{*}\left(\alpha^{*}\right)=\bar{S}\left(q_{n}^{*}\left(\alpha^{*}\right), q_{r}^{*}\left(\alpha^{*}\right)\right)>\bar{S}\left(\hat{q}_{n}\left(\alpha^{*}\right), \hat{q}_{r}\left(\alpha^{*}\right)\right)$, i.e., the slope of the PC locus takes a greater value than that of the iso-welfare contour at $\left(\hat{q}_{n}\left(\alpha^{*}\right), \hat{q}_{r}\left(\alpha^{*}\right)\right)$. This means that we can find an inner iso-welfare contour that is tangent to the locus for PC at a point $\left(\hat{q}_{n}(\hat{\alpha}), \hat{q}_{r}(\hat{\alpha})\right)$ to the left of $\left(\hat{q}_{n}\left(\alpha^{*}\right), \hat{q}_{r}\left(\alpha^{*}\right)\right)$. Therefore, $\alpha^{*}<\hat{\alpha}$ holds true, noting that $\alpha^{*}$ and $\hat{\alpha}$ are unique solutions for $0<\alpha<1$. 